JOURNAL OF THE

AMERICAN MATHEMATICAL SOCIETY

Volume 10, Number 3, July 1997, Pages 565-596

S 0894-0347(97)00237-3

\title{
QUANTUM SCHUBERT POLYNOMIALS
}

\author{
SERGEY FOMIN, SERGEI GELFAND, AND ALEXANDER POSTNIKOV
}

In this paper, we compute Gromov-Witten invariants of the flag manifold using a new combinatorial construction for its quantum cohomology ring. Our construction provides quantum analogues of the Bernstein-Gelfand-Gelfand results on the cohomology of the flag manifold, and the Lascoux-Schützenberger theory of Schubert polynomials. We also derive the quantum Monk's formula.

\section{INTRODUCTION}

Let $F l_{n}$ be the manifold of complete flags in the $n$-dimensional linear space $\mathbb{C}^{n}$. The cohomology ring $\mathrm{H}^{*}\left(F l_{n}, \mathbb{Z}\right)$ can be described in two different ways. An algebraic description due to A. Borel [5] represents it canonically as a quotient of a polynomial ring:

$$
\mathrm{H}^{*}\left(F l_{n}, \mathbb{Z}\right) \cong \mathbb{Z}\left[x_{1}, \ldots, x_{n}\right] / I_{n},
$$

where $I_{n}$ is the ideal generated by symmetric polynomials in $x_{1}, \ldots, x_{n}$ without constant term.

Another, geometric, description of the cohomology ring of the flag manifold is based on the decomposition of $F l_{n}$ into Schubert cells. These are even-dimensional cells indexed by the elements $w$ of the symmetric group $S_{n}$. The corresponding cohomology classes $\sigma_{w}$, called Schubert classes, form an additive basis in $\mathrm{H}^{*}\left(F l_{n}, \mathbb{Z}\right)$.

To relate the two descriptions, one would like to determine which elements of $\mathbb{Z}\left[x_{1}, \ldots, x_{n}\right] / I_{n}$ correspond to the Schubert classes under the isomorphism (1.1). This was first done in [2] (see also [8]) for a general case of an arbitrary complex semisimple Lie group. Later, Lascoux and Schützenberger [22] came up with a combinatorial version of this theory (for the type $A$ ) by introducing remarkable polynomial representatives of the Schubert classes $\sigma_{w}$ called Schubert polynomials and denoted $\mathfrak{S}_{w}$.

Recently, motivated by ideas that came from the string theory [31, 30], mathematicians defined, for any Kähler algebraic manifold $X$, the (small) quantum cohomology ring $\mathrm{QH}^{*}(X, \mathbb{Z})$, which is a certain deformation of the classical cohomology ring (see, e.g., $[28,19,14]$ and references therein). The additive structure of $\mathrm{QH}^{*}(X, \mathbb{Z})$ is essentially the same as that of ordinary cohomology. In particular, $\mathrm{QH}^{*}\left(F l_{n}, \mathbb{Z}\right)$ is canonically isomorphic, as an abelian group, to the tensor product $\mathrm{H}^{*}\left(F l_{n}, \mathbb{Z}\right) \otimes \mathbb{Z}\left[q_{1}, \ldots, q_{n-1}\right]$, where the $q_{i}$ are formal variables (deformation parameters). The multiplicative structure of the quantum cohomology is however

Received by the editors July 8, 1996 and, in revised form, December 23, 1996.

1991 Mathematics Subject Classification. Primary 14M15; Secondary 05E15, 14 N10.

Key words and phrases. Gromov-Witten invariants, quantum cohomology, flag manifold, Schubert polynomials.

The first author was supported in part by NSF grant DMS-9400914.

(C)1997 American Mathematical Society 
deformed compared to the classical cohomology ring $\mathrm{H}^{*}\left(F l_{n}, \mathbb{Z}\right)$, and specializes to it in the classical limit $q_{1}=\cdots=q_{n-1}=0$. The structure constants for the quantum multiplication are the 3-point Gromov-Witten invariants of genus 0. Informally, these invariants count equivalence classes of certain rational curves in the algebraic variety $F l_{n}$.

The quantum analogue of Borel's theorem was recently obtained by Givental and Kim $[15,16,17,18]$ and Ciocan-Fontanine [7]. They showed that there is a canonical ring isomorphism

$$
\mathrm{QH}^{*}\left(F l_{n}, \mathbb{Z}\right) \cong \mathbb{Z}\left[q_{1}, \ldots, q_{n-1}\right]\left[x_{1}, \ldots, x_{n}\right] / I_{n}^{q},
$$

where $I_{n}^{q}$ is the ideal generated by the coefficients $E_{1}^{n}, \ldots, E_{n}^{n}$ of the characteristic polynomial

$$
\operatorname{det}\left(1+\lambda G_{n}\right)=\sum_{i=0}^{n} E_{i}^{n} \lambda^{i}
$$

of the matrix

$$
G_{n}=\left(\begin{array}{ccccc}
x_{1} & q_{1} & 0 & \cdots & 0 \\
-1 & x_{2} & q_{2} & \cdots & 0 \\
0 & -1 & x_{3} & \cdots & 0 \\
\vdots & \vdots & \vdots & \ddots & \vdots \\
0 & 0 & 0 & \cdots & x_{n}
\end{array}\right)
$$

This result specializes to Borel's theorem in the classical limit $q_{1}=\cdots=q_{n-1}=0$, since in that case $E_{i}^{n}$ specializes to $e_{i}^{n}=e_{i}\left(x_{1}, \ldots, x_{n}\right)$, the elementary symmetric polynomial of degree $i$. We call $E_{i}^{n}$ the $i$ 'th quantum elementary polynomial in the variables $x_{1}, \ldots, x_{n}$.

In what follows, $\mathbb{Z}[q]$ stands for $\mathbb{Z}\left[q_{1}, \ldots, q_{n-1}\right]$. Analogously, $\mathbb{Z}[q, x]$ abbreviates $\mathbb{Z}\left[q_{1}, \ldots, q_{n-1}\right]\left[x_{1}, \ldots, x_{n}\right]$.

The next natural problem arising in the theory of quantum cohomology of the flag manifold is that of finding an algebraic/combinatorial method for computing the structure constants of quantum multiplication in the basis of Schubert classes (the Gromov-Witten invariants). Since the general structure of the quantum cohomology ring is given by (1.2), a solution to this problem can be obtained from an algebraic description of the elements of the quotient ring $\mathbb{Z}[q, x] / I_{n}^{q}$ that represent Schubert classes under the isomorphism (1.2). In other words, one would like to express a given Schubert class in terms of the generators $x_{i}$ using the quantum cohomology operations.

In this paper, we solve these problems, thus obtaining a quantum analogue of the Bernstein-Gelfand-Gelfand result. Our solution is essentially combinatorial, and only relies on few properties of the quantum cohomology, which can be expressed in elementary terms (see Properties 4.1-4.4).

The main question can be stated without mentioning Schubert classes. Suppose a cohomology class $c$ is written as a polynomial in the generators $x_{i}$ in the ordinary cohomology ring of the flag manifold. How can $c$ be expressed in terms of the $x_{i}$ using the quantum cohomology operations? In other words, one would like a direct algebraic description of the quantization map defined by the following commutative 
diagram:

$$
\begin{array}{ccc}
\mathrm{H}^{*}\left(F l_{n}\right) \otimes \mathbb{Z}[q] & \cong & \mathbb{Z}[q, x] / I_{n} \\
\downarrow & & \downarrow \text { quantization map } \\
\mathrm{QH}^{*}\left(F l_{n}\right) & \cong & \mathbb{Z}[q, x] / I_{n}^{q}
\end{array}
$$

In this diagram, the horizontal maps correspond to the ring isomorphisms (1.1) and (1.2); the left vertical arrow represents the tautological $\mathbb{Z}[q]$-linear map.

In order to state our main results, we need some notation. Let

$$
e_{i_{1} \ldots i_{n-1}}=e_{i_{1}}^{1} e_{i_{2}}^{2} \cdots e_{i_{n-1}}^{n-1}=e_{i_{1}}\left(x_{1}\right) e_{i_{2}}\left(x_{1}, x_{2}\right) \cdots e_{i_{n-1}}\left(x_{1}, \ldots, x_{n-1}\right),
$$

where we assume $0 \leq i_{k} \leq k$ for all $k$; by convention, $e_{0}^{k}=1$. Similarly, let

$$
E_{i_{1} \ldots i_{n-1}}=E_{i_{1}}^{1} E_{i_{2}}^{2} \cdots E_{i_{n-1}}^{n-1},
$$

where each $E_{i_{k}}^{k}$ is a quantum elementary polynomial in $x_{1}, \ldots, x_{k}$, as defined above by (1.3)-(1.4). It is not hard to show (see Section 3) that the cosets of the polynomials $e_{i_{1} \ldots i_{n-1}}$ (respectively, $E_{i_{1} \ldots i_{n-1}}$ ) form a $\mathbb{Z}[q]$-linear basis in the quotient space $\mathbb{Z}[q, x] / I_{n}$ (respectively, $\mathbb{Z}[q, x] / I_{n}^{q}$ ).

Theorem 1.1. The quantization map is the $\mathbb{Z}[q]$-linear map $\mathbb{Z}[q, x] / I_{n} \rightarrow \mathbb{Z}[q, x] / I_{n}^{q}$ that sends each coset of $e_{i_{1} \ldots i_{n-1}}$ to the corresponding coset of $E_{i_{1} \ldots i_{n-1}}$.

The quantization map can also be described in terms of a certain family of commuting difference operators (see Section 5).

In the language of Schubert polynomials, Theorem 1.1 can be restated as follows. For $w \in S_{n}$, write the uniquely defined expansion $\mathfrak{S}_{w}=\sum \alpha_{i_{1} \ldots i_{n-1}} e_{i_{1} \ldots i_{n-1}}$, with integer coefficients $\alpha_{i_{1} \ldots i_{n-1}}$. Define the quantum Schubert polynomial $\mathfrak{S}_{w}^{q}$ by

$$
\mathfrak{S}_{w}^{q}=\sum \alpha_{i_{1} \ldots i_{n-1}} E_{i_{1} \ldots i_{n-1}} .
$$

Theorem 1.2. The quantum Schubert polynomial $\mathfrak{S}_{w}^{q}$, as defined above, represents the image of the corresponding Schubert class $\sigma_{w}$ under the canonical isomorphism $\mathrm{QH}^{*}\left(F l_{n}\right) \rightarrow \mathbb{Z}[q, x] / I_{n}^{q}$.

Thus the structure constants of the quantum cohomology ring $\mathrm{QH}^{*}\left(F l_{n}\right)$ with respect to the basis of Schubert classes (i.e., the corresponding 3-point GromovWitten invariants) are equal to the structure constants of the ring $\mathbb{Z}[q, x] / I_{n}^{q}$ with respect to the basis of quantum Schubert polynomials.

An alternative approach to describing the structure of the quantum cohomology ring involves an explicit rule for the expansion of the quantum product of an arbitrary Schubert class $\sigma_{w}$ by a two-dimensional class $\sigma_{s_{r}}$, where $s_{r}$ denotes the transposition $(r r+1)$. To state this rule, we need some extra notation. For $1 \leq a<b \leq n$, let $t_{a b}=(a b) \in S_{n}$ denote the transposition of $a$ and $b$. Also, let $q_{a b}=q_{a} q_{a+1} \cdots q_{b-1}$.

Theorem 1.3 (Quantum Monk's formula). For $w \in S_{n}$ and $1 \leq r<n$, the quantum product $\sigma_{s_{r}} * \sigma_{w}$ of the Schubert classes $\sigma_{s_{r}}$ and $\sigma_{w}$ is given by

$$
\sigma_{s_{r}} * \sigma_{w}=\sum \sigma_{w t_{a b}}+\sum q_{c d} \sigma_{w t_{c d}}
$$

where the first sum is over all transpositions $t_{a b}$ such that $a \leq r<b$ and $\ell\left(w t_{a b}\right)=$ $\ell(w)+1$, and the second sum is over all transpositions $t_{c d}$ such that $c \leq r<d$ and $\ell\left(w t_{c d}\right)=\ell(w)-\ell\left(t_{c d}\right)=\ell(w)-2(d-c)+1$. 
In the classical limit $q_{1}=\cdots=q_{n-1}=0$, equation (1.8) becomes the classical Monk's formula [26] (see Theorem 2.8).

We present below the general outline of the paper. In Section 2, the necessary background is reviewed, including basic facts from the theory of classical cohomology of the flag manifolds, ordinary Schubert polynomials, quantum cohomology, and Gromov-Witten invariants. In Section 3, we study the polynomials $e_{i_{1} \ldots i_{m}}$ and their quantum counterparts $E_{i_{1} \ldots i_{m}}$. This allows us to derive some basic properties of the combinatorially defined quantum Schubert polynomials $\mathfrak{S}_{w}^{q}$, and describe a method for their computation. Section 4 is devoted to the proof of Theorem 1.2. The crucial ingredient of this proof is the orthogonality property, whose combinatorial proof is postponed until Section 6. This proof relies on a description of the quantization map that involves a family of commuting difference operators, which is given in Section 5. Section 7 contains the proof of the quantum Monk's formula. In Section 8, we review our main results. Following that, we discuss in Section 9 the problem of axiomatic characterization of the quantum Schubert polynomials. Our particular choice of polynomial representatives of Schubert classes is uniquely determined by the stability property discussed in Section 10. The quantum complete homogeneous polynomials are studied in Section 11. In Section 12, we apply Gröbner basis techniques for efficient computation of $k$-point GromovWitten invariants of the flag manifolds. Sections 13 and 14 contain the tables of Gromov-Witten invariants for the flag manifolds $\mathrm{Fl}_{3}$ and $\mathrm{Fl}_{4}$, and the tables of quantum Schubert polynomials for $S_{2}, S_{3}$, and $S_{4}$.

Among the many open problems in the field, we will only mention a few. The first natural task is to extend the theory to the case of an arbitrary root system, thus providing a quantum analogue to the corresponding result in [2]. It would be interesting to find a combinatorial construction for the quantum Schubert polynomials for other classical series (cf. $[4,11,27]$ ), and to compute the Gromov-Witten invariants of partial flag manifolds ${ }^{1}$ (cf. $\left.[1,16]\right)$.

\section{Preliminaries}

2.1. Flag manifold. We begin with reviewing the basic results on the classical cohomology of the flag manifold. Details can be found, e.g., in [13, Chapter 10].

Let $F l_{n}$ be the flag manifold whose points are the complete flags of subspaces

$$
U .=\left(U_{1} \subset U_{2} \subset \cdots \subset U_{n}=\mathbb{C}^{n}\right), \quad \operatorname{dim} U_{i}=i,
$$

in the $n$-dimensional linear space $\mathbb{C}^{n}$. The space $F l_{n}$ comes equipped with the flag of tautological vector bundles $0=\mathcal{E}_{0} \subset \mathcal{E}_{1} \subset \cdots \subset \mathcal{E}_{n-1} \subset \mathcal{E}_{n}=\mathbb{C}^{n}$; the fiber of $\mathcal{E}_{i}$ at the point $(2.1)$ is $U_{i}$.

Consider the ring homomorphism

$$
\pi: \mathbb{Z}\left[x_{1}, \ldots, x_{n}\right] \longrightarrow \mathrm{H}^{*}\left(F l_{n}, \mathbb{Z}\right)
$$

given by $\pi\left(x_{i}\right)=-c_{1}\left(\mathcal{E}_{i} / \mathcal{E}_{i-1}\right)$, where $c_{1}\left(\mathcal{E}_{i} / \mathcal{E}_{i-1}\right) \in \mathrm{H}^{2}\left(F l_{n}, \mathbb{Z}\right), i=1, \ldots, n$, is the first Chern class of the line bundle $\mathcal{E}_{i} / \mathcal{E}_{i-1}$. Let $I_{n} \subset \mathbb{Z}\left[x_{1}, \ldots, x_{n}\right]$ be the ideal generated by all symmetric polynomials without constant term - or, equivalently, by the elementary symmetric polynomials $e_{i}\left(x_{1}, \ldots, x_{n}\right)$, for $i=1, \ldots, n$. The following classical result is due to A. Borel [5].

\footnotetext{
${ }^{1}$ Note added in proof. The latest developments in "quantum Schubert calculus" are reviewed and unified in W. Fulton's recent note "Universal Schubert polynomials".
} 
Theorem 2.1. The kernel of the homomorphism $\pi$ is $I_{n}$. The induced map

$$
\mathbb{Z}\left[x_{1}, \ldots, x_{n}\right] / I_{n} \longrightarrow \mathrm{H}^{*}\left(F l_{n}, \mathbb{Z}\right)
$$

is an isomorphism.

A geometric description of $\mathrm{H}^{*}\left(F l_{n}, \mathbb{Z}\right)$ is based on a decomposition of $F l_{n}$ into even-dimensional cells indexed by the elements of the symmetric group $S_{n}$. These cells are described in terms of a relative position of a flag $U$. with respect to a fixed reference flag $V$. $\in F l_{n}$, as follows.

Let $v_{1}, \ldots, v_{n}$ be the standard basis in $\mathbb{C}^{n}$, and let $V_{b}$ denote the $b$-dimensional subspace spanned by $v_{n+1-b}, \ldots, v_{n}$. For $w \in S_{n}$, define the dual Schubert cell $\Omega_{w}^{o}$ as the set of all flags $U . \in F l_{n}$ such that, for all $a, b \in\{1, \ldots, n\}$,

$$
\operatorname{dim}\left(U_{a} \cap V_{b}\right)=\#\{1 \leq i \leq a, n+1-w(i) \leq b\} .
$$

Let $\Omega_{w}$ be the closure of $\Omega_{w}^{o}$ (the corresponding Schubert variety). The (real) dimension of $\Omega_{w}$ is $n(n-1)-2 l$, where $l=\ell(w)$ is the length of $w$ (the number of inversions). Let $\left[\Omega_{w}\right] \in \mathrm{H}_{n(n-1)-2 l}\left(F l_{n}, \mathbb{Z}\right)$ be the fundamental cycle of $\Omega_{w}$. Define the Schubert class

$$
\sigma_{w}=\left[\Omega_{w}\right]^{*} \in \mathrm{H}^{2 l}\left(F l_{n}, \mathbb{Z}\right)
$$

as the cohomology class corresponding to the fundamental cycle $\left[\Omega_{w}\right]$ under the natural isomorphism $\mathrm{H}_{n(n-1)-2 l}\left(F l_{n}, \mathbb{Z}\right) \cong \mathrm{H}^{2 l}\left(F l_{n}, \mathbb{Z}\right)$. The following result of C. Ehresmann [9] is classical.

Theorem 2.2. The Schubert classes $\sigma_{w}, w \in S_{n}$, form an additive basis in the free abelian group $\mathrm{H}^{*}\left(F l_{n}, \mathbb{Z}\right)$. Thus the rank of $\mathrm{H}^{*}\left(F l_{n}, \mathbb{Z}\right)$ is $n !$.

In particular, $\mathrm{H}^{2}\left(F l_{n}, \mathbb{Z}\right)$ is spanned by the classes $\sigma_{s_{i}}=\pi\left(x_{1}+\cdots+x_{i}\right), i=$ $1, \ldots, n-1$, where $\pi$ is defined by $(2.2)$.

2.2. Divided differences and Schubert polynomials. In [2], Bernstein, Gelfand, and Gelfand suggested a procedure, based on divided difference recurrences, that can be used to compute the elements of the quotient ring $\mathbb{C}\left[x_{1}, \ldots, x_{n}\right] / I_{n}$ which correspond to the Schubert classes. Explicit combinatorial representatives called the Schubert polynomials were then discovered by Lascoux and Schützenberger [22]. In this section, we review the main definitions and basic facts of this theory. For more details see, e.g., [25].

In the symmetric group $S_{n}$, let $s_{i}$ denote the adjacent transposition $(i i+1)$. For a permutation $w \in S_{n}$, an expression $w=s_{i_{1}} s_{i_{2}} \cdots s_{i_{l}}$ of minimal possible length $l$ is called a reduced decomposition, $l=\ell(w)$ is the length of $w$, and the sequence $i_{1}, i_{2}, \ldots, i_{l}$ is called a reduced word for $w$. For example, the transposition $t_{i j}, i<j$, that interchanges $i$ and $j$ has a reduced decomposition $t_{i j}=$ $s_{i} s_{i+1} \cdots s_{j-2} s_{j-1} s_{j-2} \cdots s_{i+1} s_{i}$, among others.

Let $f=f\left(x_{1}, \ldots, x_{n}\right)$ be a function of $x_{1}, \ldots, x_{n}$. For $w \in S_{n}$, denote $w f=$ $f\left(x_{w^{-1}(1)}, \ldots, x_{w^{-1}(n)}\right)$. The divided difference operator $\partial_{i}$ is then defined by

$$
\partial_{i} f=\left(f-s_{i} f\right) /\left(x_{i}-x_{i+1}\right) .
$$


Obviously, $\mathbb{Z}\left[x_{1}, \ldots, x_{n}\right]$ is invariant under $\partial_{i}, i=1, \ldots, n-1$. The operators $\partial_{i}$ satisfy the following relations:

$$
\begin{aligned}
& \partial_{i} \partial_{j}=\partial_{j} \partial_{i} \text { for }|i-j|>1, \\
& \partial_{i} \partial_{i+1} \partial_{i}=\partial_{i+1} \partial_{i} \partial_{i+1}, \\
& \partial_{i}^{2}=0 .
\end{aligned}
$$

For any permutation $w$, define the operator $\partial_{w}$ by $\partial_{w}=\partial_{i_{1}} \partial_{i_{2}} \cdots \partial_{i_{l}}$, where $w=$ $s_{i_{1}} s_{i_{2}} \cdots s_{i_{l}}$ is a reduced decomposition. It follows from the relations (2.3) that $\partial_{w}$ does not depend on the choice of such reduced decomposition.

The following properties of the divided differences will be used in the sequel.

Proposition $2.3([25,2.7])$. Let $v$ and $w$ be permutations. Then

$$
\partial_{v} \partial_{w}= \begin{cases}\partial_{v w} & \text { if } \ell(v w)=\ell(v)+\ell(w), \\ 0 & \text { otherwise. }\end{cases}
$$

Proposition 2.4 ([25, (2.2), 2.13] "Leibniz formula"). 1. For any polynomials $f$ and $g$ and any $i$,

$$
\partial_{i}(f g)=\partial_{i}(f) \cdot g+\left(s_{i} f\right)\left(\partial_{i} g\right) .
$$

In particular, $\partial_{i}$ commutes with multiplication by any polynomial which is symmetric in $x_{i}$ and $x_{i+1}$.

2. For a linear form $f=\sum \lambda_{i} x_{i}$, we have

$$
\partial_{w}(f g)=w(f) \partial_{w} g+\sum\left(\lambda_{i}-\lambda_{j}\right) \partial_{w t_{i j}} g,
$$

where the sum is over all $i<j$ such that $\ell\left(w t_{i j}\right)=\ell(w)-1$.

Let $\delta=\delta_{n}=(n-1, n-2, \ldots, 1,0)$ and $x^{\delta}=x_{1}^{n-1} x_{2}^{n-2} \cdots x_{n-1}$. For a permutation $w \in S_{n}$, the Schubert polynomial $\mathfrak{S}_{w}$ of Lascoux and Schützenberger is defined by $\mathfrak{S}_{w}=\partial_{w^{-1} w_{\mathrm{o}}} x^{\delta}$, where $w_{\mathrm{o}}$ is the longest element in $S_{n}$, given by $w_{\mathrm{o}}(i)=n+1-i$. Equivalently,

$$
\mathfrak{S}_{w_{\mathrm{o}}}=x^{\delta} \quad \text { and } \quad \mathfrak{S}_{w s_{i}}=\partial_{i} \mathfrak{S}_{w} \quad \text { whenever } \quad \ell\left(w s_{i}\right)=\ell(w)-1 .
$$

The following fundamental result is an immediate corollary of [2].

Theorem 2.5. The Schubert polynomials represent Schubert classes, i.e., in the notation of (2.2) and Theorem 2.1, $\pi\left(\mathfrak{S}_{w}\right)=\sigma_{w}$.

Let $L_{n}$ denote the $\mathbb{Z}$-span of the monomials $x_{1}^{a_{1}} \cdots x_{n-1}^{a_{n-1}}$ satisfying $0 \leq a_{k} \leq$ $n-k$. It is easy to see that $L_{n}$ is invariant under each of the operators $\partial_{1}, \ldots, \partial_{n-1}$. This leads to the following result.

Proposition $2.6([22,25])$. The space $L_{n}$ is complementary to the ideal $I_{n}$. The Schubert polynomials $\mathfrak{S}_{w}$, for $w \in S_{n}$, form a linear basis of $L_{n}$.

We will also need the following properties of the Schubert polynomials.

Corollary $2.7([25,(4.2)])$. Let $v, w \in S_{n}$. Then

$$
\partial_{v} \mathfrak{S}_{w}= \begin{cases}\mathfrak{S}_{w v^{-1}} & \text { if } \ell\left(w v^{-1}\right)=\ell(w)-\ell(v), \\ 0 & \text { otherwise. }\end{cases}
$$


Theorem 2.8 (Monk's formula [26, 25]; cf. also Chevalley [6]). We have

$$
\mathfrak{S}_{s_{r}} \mathfrak{S}_{w}=\sum \mathfrak{S}_{w t_{i j}}
$$

where the sum is over all transpositions $t_{i j}$ such that $i \leq r<j$ and $\ell\left(w t_{i j}\right)=$ $\ell(w)+1$.

Note that $\mathfrak{S}_{s_{r}}=x_{1}+\cdots+x_{r}$.

The Schubert polynomials have the following orthogonality property (see, e.g., $[25,(5.4)])$. For a polynomial $f$, define

$$
\langle f\rangle=\left(\partial_{w_{\mathrm{o}}}(f)\right)(0, \ldots, 0) ;
$$

observe that

$$
\partial_{w_{\mathrm{o}}}(f)=\sum_{w}(-1)^{\ell(w)} w f \cdot \prod_{i<j}\left(x_{i}-x_{j}\right)^{-1} .
$$

Theorem 2.9. For $u, v \in S_{n}$,

$$
\left\langle\mathfrak{S}_{u} \mathfrak{S}_{v}\right\rangle= \begin{cases}1 & \text { if } v=w_{\mathrm{o}} u \\ 0 & \text { otherwise }\end{cases}
$$

Geometrically, $\langle f\rangle$ is the coefficient of $\sigma_{w_{\mathrm{o}}}$ in the expansion of $\pi(f) \in \mathrm{H}^{*}\left(F l_{n}\right)$ in the basis of Schubert classes $\sigma_{w}$; here $\pi$ denotes the canonical homomorphism (2.2). Theorem 2.9 can be restated as saying that the bases $\left\{\sigma_{w}\right\}$ and $\left\{\sigma_{w_{\mathrm{o}} w}\right\}$ are dual to each other with respect to the Poincare pairing in $\mathrm{H}^{*}\left(F l_{n}\right)$.

2.3. Gromov-Witten invariants and quantum cohomology. In this section, we reproduce the definitions of the Gromov-Witten invariants and the quantum cohomology ring of the flag manifold. See $[1,3,7,10,14,15,16,17,19,20,24,28]$ for details, and for various approaches to the subject.

The homology classes $\left[\Omega_{w_{0} s_{i}}\right], i=1, \ldots, n-1$, of two-dimensional Schubert varieties form a linear basis in $\mathrm{H}_{2}\left(F l_{n}, \mathbb{Z}\right)$. An algebraic map $f: \mathbb{P}^{1} \rightarrow F l_{n}$ has multidegree $d=\left(d_{1}, \ldots, d_{n-1}\right)$ if $f_{*}\left[\mathbb{P}^{1}\right]=\sum d_{i}\left[\Omega_{w_{\mathrm{o}} s_{i}}\right]$. The moduli space $\mathcal{M}_{d}\left(\mathbb{P}^{1}, F l_{n}\right)$ of such maps is a smooth algebraic variety of (complex) dimension

$$
\mathcal{D}=\left(\begin{array}{l}
n \\
2
\end{array}\right)+2 \sum_{i=1}^{n-1} d_{i} .
$$

For a subvariety $Y \subset F l_{n}$ and a point $t \in \mathbb{P}^{1}$, let us denote

$$
Y(t)=\left\{f \in \mathcal{M}_{d}\left(\mathbb{P}^{1}, F l_{n}\right) \mid f(t) \in Y\right\} .
$$

The codimension of $Y(t)$ in $\mathcal{M}_{d}\left(\mathbb{P}^{1}, F l_{n}\right)$ equals the codimension of $Y$ in $F l_{n}$.

Let $w_{1}, \ldots, w_{N} \in S_{n}$. The Gromov-Witten invariant of genus 0 associated to the classes $\sigma_{w_{1}}, \ldots, \sigma_{w_{N}}$ is defined as follows. Let $g_{1}, \ldots, g_{N}$ be generic elements of $G L_{n}$, and let $t_{1}, \ldots, t_{N}$ be distinct points in $\mathbb{P}^{1}$. Define

$$
\left\langle\sigma_{w_{1}}, \ldots, \sigma_{w_{N}}\right\rangle_{d}= \begin{cases}\text { number of points in } \bigcap\left(g_{i} \Omega_{w_{i}}\right)\left(t_{i}\right) & \text { if } \sum \ell\left(w_{i}\right)=\mathcal{D}, \\ 0 & \text { otherwise. }\end{cases}
$$

These cardinalities are finite and independent of the choice of points $t_{i} \in \mathbb{P}^{1}$ and generic elements $g_{i} \in G L_{n}$.

Informally, the Gromov-Witten invariant $\left\langle\sigma_{w_{1}}, \ldots, \sigma_{w_{N}}\right\rangle_{d}$ of the flag manifold $F l_{n}$ counts rational curves in $F l_{n}$ which have multidegree $d=\left(d_{1}, \ldots, d_{n-1}\right)$ and 
pass through Schubert varieties $\Omega_{w_{1}}, \ldots, \Omega_{w_{N}}$. The condition $\sum \ell\left(w_{i}\right)=\mathcal{D}$ ensures that this cardinality is finite.

We will now define the (small) quantum cohomology ring $\mathrm{QH}^{*}\left(F l_{n}, \mathbb{Z}\right)$ of the flag manifold $F l_{n}$. As an abelian group,

$$
\mathrm{QH}^{*}\left(F l_{n}, \mathbb{Z}\right)=\mathrm{H}^{*}\left(F l_{n}, \mathbb{Z}\right) \otimes \mathbb{Z}\left[q_{1}, \ldots, q_{n-1}\right],
$$

where $q_{1}, \ldots, q_{n-1}$ are formal parameters. The multiplication in $\mathrm{QH}^{*}\left(F l_{n}, \mathbb{Z}\right)$ (the quantum multiplication) is a linear over $\mathbb{Z}[q]=\mathbb{Z}\left[q_{1}, \ldots, q_{n-1}\right]$ binary operation $*$ defined by

$$
\sigma_{u} * \sigma_{v}=\sum_{w \in S_{n}} \sum_{d} q^{d}\left\langle\sigma_{u}, \sigma_{v}, \sigma_{w}\right\rangle_{d} \sigma_{w_{\mathrm{o}} w},
$$

where where we denote $q^{d}=q_{1}^{d_{1}} \cdots q_{n-1}^{d_{n-1}}$ for $d=\left(d_{1}, \ldots, d_{n-1}\right)$.

Quantum multiplication is commutative and - miraculously — associative [28, 24]. The specialization $q_{1}=\cdots=q_{n-1}=0$ recovers the ordinary cohomology ring $\mathrm{H}^{*}\left(F l_{n}, \mathbb{Z}\right)$. Indeed, an algebraic map $\mathbb{P}^{1} \rightarrow F l_{n}$ of multidegree $(0, \ldots, 0)$ is constant, so the Gromov-Witten invariants $\left\langle\sigma_{u}, \sigma_{v}, \sigma_{w}\right\rangle_{(0, \ldots, 0)}$ are the usual intersection numbers.

Note that $\left\langle\sigma_{u}, \sigma_{v}, \sigma_{w}\right\rangle_{d}$ vanishes unless $\ell(u)+\ell(v)=\ell\left(w_{\mathrm{o}} w\right)+2 \sum d_{i}$ (cf. (2.10) and $(2.12))$. Thus quantum multiplication respects the grading defined by $\operatorname{deg}\left(\sigma_{w}\right)$ $=\ell(w)$ and $\operatorname{deg}\left(q_{i}\right)=2$.

The following description of the quantum cohomology ring of the flag manifold was given by Givental and Kim [15], and further justified by Kim [16, 17] and Ciocan-Fontanine [7]. Let $I_{n}^{q}$ be the ideal in the ring $\mathbb{Z}[q, x]$ that is generated by the coefficients $E_{1}^{n}, \ldots, E_{n}^{n}$ of the characteristic polynomial (1.3) of the matrix $G_{n}$ given by (1.4).

Define the $\mathbb{Z}[q]$-linear ring homomorphism

$$
\pi^{q}: \mathbb{Z}[q, x] \longrightarrow \mathrm{QH}^{*}\left(F l_{n}, \mathbb{Z}\right)
$$

by setting $\pi^{q}\left(x_{1}+\cdots+x_{i}\right)=\sigma_{s_{i}}$.

Theorem $2.10([15,16,17,7])$. The kernel of $\pi^{q}$ is $I_{n}^{q}$. The induced map

$$
\mathbb{Z}[q, x] / I_{n}^{q} \longrightarrow \mathrm{QH}^{*}\left(F l_{n}, \mathbb{Z}\right)
$$

is a ring isomorphism.

\section{QUANTIZATION VIA STANDARD MONOMIALS}

3.1. Straightening. Let $e_{i}^{k}=e_{i}\left(x_{1}, \ldots, x_{k}\right)$ be the $i$ 'th elementary symmetric polynomial:

$$
e_{i}^{k}=\sum_{1 \leq r_{1}<\cdots<r_{i} \leq k} x_{r_{1}} \cdots x_{r_{i}} .
$$

By convention, $e_{0}^{k}=1$ for $k \geq 0$, and $e_{i}^{k}=0$ unless $0 \leq i \leq k$.

The polynomials $e_{i}^{k}$ satisfy the obvious recurrence

$$
e_{i}^{k}=e_{i}^{k-1}+x_{k} e_{i-1}^{k-1} .
$$

Lemma 3.1. For $k \neq l$, we have $\partial_{l} e_{i}^{k}=0$. Moreover, $\partial_{l}$ commutes with multiplication by $e_{i}^{k}$, provided $k \neq l$. Also, $\partial_{k} e_{i}^{k}=e_{i-1}^{k-1}$.

Proof. The proof follows from Proposition 2.4, part 1. 
Lemma 3.2. For $i, j, k \geq 1$, the following relations hold:

$$
\begin{gathered}
\left(e_{i}^{k+1}-e_{i}^{k}\right) e_{j-1}^{k}=\left(e_{j}^{k+1}-e_{j}^{k}\right) e_{i-1}^{k}, \\
e_{i}^{k} e_{j}^{k}=e_{i}^{k+1} e_{j}^{k}+\sum_{l \geq 1} e_{i-l}^{k+1} e_{j+l}^{k}-\sum_{l \geq 1} e_{i-l}^{k} e_{j+l}^{k+1} .
\end{gathered}
$$

Proof. By (3.1), we have $\left(e_{i}^{k+1}-e_{i}^{k}\right) e_{j-1}^{k}=x_{k+1} e_{i-1}^{k} e_{j-1}^{k}=\left(e_{j}^{k+1}-e_{j}^{k}\right) e_{i-1}^{k}$. Equation (3.3) follows from (3.2) by induction on $i$ :

$$
\begin{aligned}
e_{j}^{k}\left(e_{i+1}^{k}-e_{i+1}^{k+1}\right) & =-e_{i}^{k}\left(e_{j+1}^{k+1}-e_{j+1}^{k}\right) \\
& =e_{i}^{k+1} e_{j+1}^{k}-e_{j+1}^{k+1} e_{i}^{k}+e_{j+1}^{k}\left(e_{i}^{k}-e_{i}^{k+1}\right) \\
& =e_{i}^{k+1} e_{j+1}^{k}-e_{j+1}^{k+1} e_{i}^{k}+\sum_{l \geq 1}\left(e_{i-l}^{k+1} e_{j+1+l}^{k}-e_{j+1+l}^{k+1} e_{i-l}^{k}\right) \\
& =\sum_{l \geq 1}\left(e_{i+1-l}^{k+1} e_{j+l}^{k}-e_{j+1}^{k+1} e_{i+1-l}^{k}\right) .
\end{aligned}
$$

For $i_{1}, \ldots, i_{m}$ such that $0 \leq i_{k} \leq k$, let

$$
e_{i_{1} \ldots i_{m}}=e_{i_{1}}^{1} \cdots e_{i_{m}}^{m} .
$$

We will call $e_{i_{1} \ldots i_{m}}$ a standard elementary monomial. (These polynomials were originally introduced in [22], and were denoted $P_{I}$ there.) In other words, a standard elementary monomial is any product of the $e_{i}^{k}$ without repetitions of upper indices $k$. Note that appending zeroes at the end of the sequence $i_{1}, \ldots, i_{m}$ does not change the standard elementary monomial.

Proposition 3.3 (Straightening). The standard elementary monomials form a linear basis in the ring $\mathbb{Z}\left[x_{1}, x_{2}, \ldots\right]$ of polynomials in infinitely many variables.

Proof. We will first show that every polynomial $f \in \mathbb{Z}\left[x_{1}, x_{2}, \ldots\right]$ belongs to the span of standard elementary monomials. Note that $x_{i}=e_{1}^{i}-e_{1}^{i-1}$; hence $f$ is a linear combination of some monomials in the $e_{i}^{k}$. Choose such a linear combination and apply to it the following straightening algorithm.

Suppose that some monomial in this linear combination is not standard. Assume it contains $e_{i}^{k} e_{j}^{k}$, with the smallest possible value of $k$. Then replace $e_{i}^{k} e_{j}^{k}$ by the right-hand side of (3.3). Because of our choice of $k$, we will not create a new repetition of upper indices with a smaller $k$. If there still are nonstandard monomials, repeat the same procedure. This process will terminate, since the total degree of the polynomial does not change. As a result, we will express $f$ as a linear combination of standard elementary monomials.

Now let us show that all standard elementary monomials are linearly independent. For suppose not. Find a nontrivial linear relation $R$ with terms of minimal possible degree. Let $k$ be the minimal index such that some $e_{i}^{k}, i>0$, appears in some monomial in $R$. By Lemma 3.1, $\partial_{k}$ annihilates every monomial not containing $e_{i}^{k}, i>0$, whereas $\partial_{k} e_{i}^{k} e_{j}^{k+1} \cdots=e_{i-1}^{k-1} e_{j}^{k+1} \cdots$. Hence applying $\partial_{k}$ to $R$ results in a nontrivial linear relation with terms of smaller degree. This contradicts the choice of $R$.

Proposition 3.3 can be used to prove the following basic result. Recall that $I_{n}$ denotes the ideal generated by the polynomials $e_{1}^{n}, \ldots, e_{n}^{n}$. 
Proposition 3.4 (see [22], [23, (2.6)-(2.7)], [25, (4.13)]). Each of the following form a $\mathbb{Z}$-linear basis in $\mathbb{Z}\left[x_{1}, \ldots, x_{n}\right] / I_{n}$ :

- the monomials $x_{1}^{a_{1}} \cdots x_{n-1}^{a_{n-1}}$ such that $0 \leq a_{k} \leq n-k$;

- the standard elementary monomials $e_{i_{1} i_{2} \ldots i_{n-1}}$;

- the Schubert polynomials $\mathfrak{S}_{w}$ for $w \in S_{n}$.

Moreover, each of these families spans the same vector space $L_{n} \subset \mathbb{Z}\left[x_{1}, \ldots, x_{n}\right]$, which is complementary to $I_{n}$.

Proof. In view of Proposition 2.6, we only need to show that the standard elementary monomials $e_{i_{1} i_{2} \ldots i_{n-1}}$ are linearly independent (this is true by Proposition 3.3) and span the same space as the monomials $x_{1}^{a_{1}} \cdots x_{n-1}^{a_{n-1}}$ satisfying $0 \leq a_{k} \leq n-k$. Indeed, each $e_{i_{1} i_{2} \ldots i_{n-1}}$ is obviously a linear combination of such monomials, and the result follows by a dimension argument.

3.2. Quantum elementary polynomials. Recall that the quantum elementary polynomial $E_{i}^{k}$ is defined as the coefficient of $\lambda^{i}$ in the characteristic polynomial $\operatorname{det}\left(1+\lambda G_{k}\right)$ of the matrix $G_{k}$ given by

$$
G_{k}=\left(\begin{array}{ccccc}
x_{1} & q_{1} & 0 & \cdots & 0 \\
-1 & x_{2} & q_{2} & \cdots & 0 \\
0 & -1 & x_{3} & \cdots & 0 \\
\vdots & \vdots & \vdots & \ddots & \vdots \\
0 & 0 & 0 & \cdots & x_{k}
\end{array}\right)
$$

By convention, $E_{i}^{k}=0$ unless $0 \leq i \leq k$.

The quantum elementary polynomials $E_{i}^{k}$ have the following combinatorial interpretation. Let us view each variable $x_{j}$ as a singleton $\{j\}, 1 \leq j \leq k$, and each $q_{r}$ as a "dimer" $\{r, r+1\}, 1 \leq r \leq k-1$. Then $E_{i}^{k}$ is the sum of all monomials in the $x_{j}$ and $q_{r}$ which correspond to disjoint collections of singletons and dimers covering exactly $i$ distinct nodes. The number of monomials in $E_{k}^{k}$ is thus equal to the $k^{\prime}$ th Fibonacci number. Also immediate from this description is the recurrence (see [15])

$$
E_{i}^{k}=E_{i}^{k-1}+x_{k} E_{i-1}^{k-1}+q_{k-1} E_{i-2}^{k-2},
$$

for any $1 \leq i \leq k$, where we assume $q_{0}=0$.

The polynomials $E_{i}^{k}$ are homogeneous with respect to the grading $\operatorname{deg}\left(x_{i}\right)=1$, $\operatorname{deg}\left(q_{j}\right)=2$, and specialize to the $e_{i}^{k}$ in the case $q_{1}=\cdots=q_{n-1}=0$.

The following analogue of (3.2) can be used for "quantum straightening."

Lemma 3.5. For $k \geq j \geq 0, k \geq i \geq 0$,

$$
E_{i}^{k} E_{j+1}^{k+1}+E_{i+1}^{k} E_{j}^{k}+q_{k} E_{i-1}^{k-1} E_{j}^{k}=E_{j}^{k} E_{i+1}^{k+1}+E_{j+1}^{k} E_{i}^{k}+q_{k} E_{j-1}^{k-1} E_{i}^{k} .
$$

Proof. By (3.5), one has

$$
E_{i}^{k}\left(E_{j+1}^{k+1}-E_{j+1}^{k}\right)=E_{i}^{k}\left(x_{k+1} E_{j}^{k}+q_{k} E_{j-1}^{k-1}\right)
$$

and

$$
E_{j}^{k}\left(E_{i+1}^{k+1}-E_{i+1}^{k}\right)=E_{j}^{k}\left(x_{k+1} E_{i}^{k}+q_{k} E_{i-1}^{k-1}\right) .
$$

Subtracting the second equation from the first, we obtain (3.6). 
By analogy with (3.4), we define a quantum standard elementary monomial by

$$
E_{i_{1} \ldots i_{m}}=E_{i_{1}}^{1} \cdots E_{i_{m}}^{m}
$$

where $0 \leq i_{k} \leq k$ for $k=1, \ldots, m$.

The following quantum analogue of Proposition 3.4 can be proved in the same way as the latter, using a straightening procedure based on Lemma 3.5.

Proposition 3.6. Each of the following form a $\mathbb{Z}[q]$-linear basis in $\mathbb{Z}[q, x] / I_{n}^{q}$ :

- the monomials $x_{1}^{a_{1}} \cdots x_{n-1}^{a_{n-1}}$ such that $0 \leq a_{k} \leq n-k$;

- the quantum standard elementary monomials $E_{i_{1} i_{2} \ldots i_{n-1}}$.

Moreover, each of these two families spans the same vector space $L_{n}^{q} \subset \mathbb{Z}[q, x]$ complementary to $I_{n}^{q}$.

Quantum straightening can be used to compute the expansion of a product of several quantum standard elementary monomials in the basis $\left\{E_{i_{1} i_{2} \ldots i_{n-1}}\right\}$ of the $\operatorname{ring} \mathbb{Z}[q, x] / I_{n}^{q}$.

3.3. Quantum Schubert polynomials. Let us recall the combinatorial definition of the quantum Schubert polynomials $\mathfrak{S}_{w}^{q}$ given in the introduction. By Proposition 3.4, one can uniquely expand an ordinary Schubert polynomial $\mathfrak{S}_{w}, w \in S_{n}$, as a linear combination of standard elementary monomials, with integer coefficients: $\mathfrak{S}_{w}=\sum \alpha_{i_{1} \ldots i_{n-1}} e_{i_{1} \ldots i_{n-1}}$. We then define

$$
\mathfrak{S}_{w}^{q}=\sum \alpha_{i_{1} \ldots i_{n-1}} E_{i_{1} \ldots i_{n-1}} .
$$

Propositions 3.4 and 3.6 immediately imply the following result.

Proposition 3.7. The quantum Schubert polynomials $\mathfrak{S}_{w}^{q}$ for $w \in S_{n}$, form a $\mathbb{Z}[q]$-linear basis in the space $L_{n}^{q}$ spanned by the monomials $x_{1}^{a_{1}} \cdots x_{n-1}^{a_{n-1}}$ satisfying $0 \leq a_{k} \leq n-k$. The $I_{n}^{q}$-cosets of these quantum Schubert polynomials form a $\mathbb{Z}[q]$-linear basis in $\mathbb{Z}[q, x] / I_{n}^{q}$.

The expansions of Schubert polynomials for $S_{n}$ in terms of the standard monomials can be computed recursively top-down, starting from $\mathfrak{S}_{w_{\mathrm{o}}}=e_{12 \ldots n-1}$. Namely, use the basic recurrence (2.6) together with the rule for computing a divided difference of an elementary symmetric polynomial (Lemma 3.1), the Leibniz formula (2.4), and the straightening procedure from Section 3.1. For example, in $S_{4}$ we have:

$$
\begin{aligned}
& \mathfrak{S}_{4321}=\mathfrak{S}_{w_{\mathrm{o}}}=e_{123} \\
& \mathfrak{S}_{3421}=\partial_{1} \mathfrak{S}_{4321}=\partial_{1} e_{123}=e_{023} \partial_{1} e_{1}^{1}=e_{023}, \\
& \mathfrak{S}_{3412}=\partial_{3} \mathfrak{S}_{3421}=\partial_{3} e_{023}=e_{020} \partial_{3} e_{3}^{3}=\left(e_{2}^{2}\right)^{2}=e_{022}-e_{013} .
\end{aligned}
$$

The corresponding quantum Schubert polynomials $\mathfrak{S}_{w}^{q}$ are then obtained by replacing each standard elementary monomial by its quantum analogue. For instance,

$$
\begin{aligned}
\mathfrak{S}_{3412}^{q}= & E_{022}-E_{013} \\
= & \left(x_{1} x_{2}+q_{1}\right)\left(x_{1} x_{2}+x_{1} x_{3}+x_{2} x_{3}+q_{1}+q_{2}\right) \\
& -\left(x_{1}+x_{2}\right)\left(x_{1} x_{2} x_{3}+q_{1} x_{3}+q_{2} x_{1}\right) \\
= & x_{1}^{2} x_{2}^{2}+2 q_{1} x_{1} x_{2}-q_{2} x_{1}^{2}+q_{1}^{2}+q_{1} q_{2} .
\end{aligned}
$$




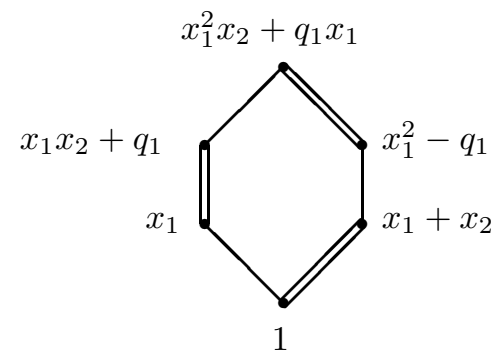

Figure 1. Quantum Schubert polynomials for $S_{3}$

In Section 14, we provide the tables of quantum Schubert polynomials for $S_{2}$, $S_{3}$ (cf. Figure 1), and $S_{4}$. For each permutation $w$, we also give the expansion of the Schubert polynomial $\mathfrak{S}_{w}$ as a linear combination of standard elementary monomials.

Proposition 3.8. $\mathfrak{S}_{w}^{q}$ is a homogeneous polynomial of degree $\ell(w)$, with respect to the grading defined by $\operatorname{deg}\left(x_{i}\right)=1$ and $\operatorname{deg}\left(q_{j}\right)=2$. Specializing $q_{1}=\cdots=q_{n-1}=$ 0 yields $\mathfrak{S}_{w}^{q}=\mathfrak{S}_{w}$, the classical Schubert polynomials.

Proof. The proof follows from the corresponding properties of quantum elementary polynomials.

Proposition 3.8 implies that the transition matrices between the bases $\left\{\mathfrak{S}_{w}^{q}\right\}$ and $\left\{\mathfrak{S}_{w}\right\}$ are unipotent triangular, with respect to any linear ordering that is consistent with the length function $\ell(w)$.

To conclude this section, we formulate the quantum analogue of the orthogonality property (2.9) of the Schubert polynomials. Similarly to the classical case, orthogonality of Schubert classes is a trivial consequence of the quantum cohomology definitions (cf. Property 9.4 below). At this point, however, we have not proved yet that our combinatorially defined quantum Schubert polynomials $\mathfrak{S}_{w}^{q}$ represent Schubert classes $\sigma_{w}$ in the quantum cohomology ring. Moreover, the proof of this fact given in Section 4 depends on a combinatorial proof of the orthogonality of the $\mathfrak{S}_{w}^{q}$.

For $F \in \mathbb{Z}[q, x] / I_{n}^{q}$, let $\left\langle\langle F\rangle \in \mathbb{Z}[q]\right.$ denote the coefficient of $\mathfrak{S}_{w_{\mathrm{o}}}^{q}$ in the expansion of $F$ in the basis of quantum Schubert polynomials (cf. Proposition 3.7). Equivalently, $\left\langle\langle F\rangle\right.$ is the coefficient of the staircase monomial $x^{\delta}=x_{1}^{n-1} x_{2}^{n-2} \cdots x_{n-1}$ in the monomial expansion of $F$.

The following result is the quantum analogue of Theorem 2.9. Its proof is postponed until Section 6.

Theorem 3.9 (Orthogonality). For $u, v \in S_{n}$,

$$
\left\langle\left\langle\mathfrak{S}_{u}^{q} \mathfrak{S}_{v}^{q}\right\rangle= \begin{cases}1 & \text { if } v=w_{\mathrm{o}} u \\ 0 & \text { otherwise. }\end{cases}\right.
$$

\section{Proof of Theorems 1.1 And 1.2}

We begin with an outline of the proof. Let $\left\{\mathcal{Q}_{w}\right\}$ be the "geometric" quantum Schubert polynomials, i.e., the elements of the quotient $\mathbb{Z}[q, x] / I_{n}^{q}$ which represent 
the Schubert classes under the isomorphism (2.15). Theorem 1.2 can be reformulated as saying that the $\mathcal{Q}_{w}$ coincide with the cosets of the combinatorially defined polynomials $\mathfrak{S}_{w}^{q}$.

To prove Theorem 1.2 (thus Theorem 1.1), we will need four particular properties of the elements $\mathcal{Q}_{w}$ (see Properties 4.1-4.4). The first three of these properties easily follow from the definition of quantum cohomology; the fourth one is a theorem of Ciocan-Fontanine [7]. We will use these properties of the $\mathcal{Q}_{w}$, in conjunction with several properties of the polynomials $\mathfrak{S}_{w}^{q}$ (most notably, their orthogonality, Theorem 3.9), to demonstrate that the $\mathcal{Q}_{w}$ coincide with the cosets of the $\mathfrak{S}_{w}^{q}$. As a byproduct, this will immediately imply that Properties 4.1-4.4 uniquely determine the $\mathcal{Q}_{w}$. The issue of axiomatic characterization of the quantum Schubert polynomials is discussed in Section 9.

In this section, we work in the quotient ring $\mathbb{Z}[q, x] / I_{n}^{q}$. All polynomials should be understood as representing cosets modulo $I_{n}^{q}$.

Property 4.1 (Homogeneity). Every $\mathcal{Q}_{w}$ is homogeneous of degree $\ell(w)$, assuming $\operatorname{deg}\left(x_{i}\right)=1$ and $\operatorname{deg}\left(q_{j}\right)=2$.

Proof. See the remark following (2.14).

Property 4.2 (Classical limit). Specializing $q_{1}=\cdots=q_{n-1}=0$ yields $\mathcal{Q}_{w}=$ $\mathfrak{S}_{w}$.

Proof. This reflects the fact that this specialization converts the quantum cohomology of $F l_{n}$ into the usual one.

Properties 4.1 and 4.2 imply that the $\mathcal{Q}_{w}$ form a $\mathbb{Z}[q]$-linear basis in $\mathbb{Z}[q, x] / I_{n}^{q}$, and the transition matrices between any two of the bases $\left\{\mathcal{Q}_{w}\right\},\left\{\mathfrak{S}_{w}^{q}\right\}$, and $\left\{\mathfrak{S}_{w}\right\}$ are unipotent triangular, with respect to any linear ordering consistent with $\ell(w)$.

The next property is a reformulation of the fact that the Gromov-Witten invariants are nonnegative integers.

Property 4.3 (Nonnegativity of structure constants). The structure constants of the ring $\mathbb{Z}[q, x] / I_{n}^{q}$, with respect to the basis $\left\{\mathcal{Q}_{w}\right\}$, are polynomials in $q_{1}, \ldots, q_{n-1}$ with nonnegative integer coefficients.

Let $\mathbb{Z}_{+}[q]$ be the set of all polynomials in the $q_{j}$ whose coefficients are nonnegative integers. Let $\mathrm{QH}_{+}^{*}$ be the set of all linear combinations of the $\mathcal{Q}_{w}$ with coefficients in $\mathbb{Z}_{+}[q]$. By Property 4.3, $\mathrm{QH}_{+}^{*}$ is a semiring, i.e., is closed under addition and multiplication.

As a corollary, $\left\langle\left\langle\mathcal{Q}_{w_{1}} \cdots \mathcal{Q}_{w_{k}}\right\rangle\right\rangle \mathbb{Z}_{+}[q]$, for any $w_{1}, \ldots, w_{k} \in S_{n}$, where $\langle\langle\cdots\rangle$ is defined as in Section 3.3. Indeed, $\left\langle\left\langle\mathcal{Q}_{w_{1}} \cdots \mathcal{Q}_{w_{k}}\right\rangle\right\rangle$ is equal to the coefficient of $\mathcal{Q}_{w_{\circ}}$ in the expansion of this product in the basis $\left\{\mathcal{Q}_{w}\right\}$, since the transition matrix between $\left\{\mathcal{Q}_{w}\right\}$ and $\left\{\mathfrak{S}_{w}\right\}$ is unipotent triangular.

It is well known that the ordinary Schubert polynomial $\mathfrak{S}_{w}$ for a cycle $w=$ $s_{k-i+1} \cdots s_{k}$ is the elementary symmetric polynomial $e_{i}^{k}$. The following result, which is a restatement of formula (3) in [7], provides a quantum analogue to this fact.

Property 4.4 (Quantum elementary polynomials). For a cycle $w=s_{k-i+1} \cdots s_{k}$ $\in S_{n}$, the polynomial $\mathcal{Q}_{w}$ is $E_{i}^{k}$, the quantum elementary polynomial.

In our proof of Theorem 1.2, we will only need the following corollary of this property: every quantum elementary polynomial $E_{i}^{k}$ belongs to the semiring $\mathrm{QH}_{+}^{*}$. 
It then follows by Property 4.3 that every quantum standard elementary monomial belongs to $\mathrm{QH}_{+}^{*}$.

We are now prepared to give a proof of Theorem 1.2. Fix a nonnegative integer $l \leq \ell\left(w_{\mathrm{o}}\right)$. By Proposition 3.4, the polynomials $\mathfrak{S}_{w}, \ell(w)=l$, are related to the $e_{i_{1} \ldots i_{n-1}}, i_{1}+\cdots+i_{n-1}=l$, by a nondegenerate linear transformation. Moreover, each $e_{i_{1} \ldots i_{n-1}}$ is a nonnegative integer combination of the $\mathfrak{S}_{w}$, since $e_{i_{1} \ldots i_{n-1}}$ is a product of Schubert polynomials $e_{i_{k}}^{k}$, and the classical structure constants are nonnegative. Every $\mathfrak{S}_{w}, \ell(w)=l$, should enter the expansion of at least one $e_{i_{1} \ldots i_{n-1}}, i_{1}+\cdots+i_{n-1}=l$. Therefore

$$
\sum_{i_{1}+\cdots+i_{n-1}=l} e_{i_{1} \ldots i_{n-1}}=\sum_{\ell(w)=l} \alpha_{w} \mathfrak{S}_{w},
$$

with certain positive $\alpha_{w}$. Using the definition (3.8) of the quantum Schubert polynomials and the fact that $E_{i_{1} \ldots i_{n-1}} \in \mathrm{QH}_{+}^{*}$, we obtain:

$$
\sum_{\ell(w)=l} \alpha_{w} \mathfrak{S}_{w}^{q} \in \mathrm{QH}_{+}^{*} .
$$

By Properties 4.1 and 4.2 , each $\mathfrak{S}_{w}^{q}$ is equal to $\mathcal{Q}_{w}$ plus a $\mathbb{Z}[q]$-linear combination of some $\mathcal{Q}_{v}$ with $\ell(v)<\ell(w)$. It follows that

$$
\sum_{\ell(w)=l} \alpha_{w} \mathfrak{S}_{w}^{q}=\sum_{\ell(w)=l} \alpha_{w} \mathcal{Q}_{w}+\left\langle\text { linear combination of } \mathcal{Q}_{v} \text { with } \ell(v)<\ell(w)\right\rangle,
$$

and (4.1) yields

$$
\sum_{\ell(w)=l} \alpha_{w}\left(\mathfrak{S}_{w}^{q}-\mathcal{Q}_{w}\right) \in \mathrm{QH}_{+}^{*} .
$$

Let $j_{1}, \ldots, j_{n-1}$ be such that

$$
j_{1}+\cdots+j_{n-1}>\ell\left(w_{\mathrm{o}}\right)-l .
$$

Since $E_{j_{1} \ldots j_{n-1}} \in \mathrm{QH}_{+}^{*}$, Property 4.3 implies that, for any $w$,

$$
\left.\left\langle E_{j_{1} \ldots j_{n-1}} \mathcal{Q}_{w}\right\rangle\right\rangle \in \mathbb{Z}_{+}[q] .
$$

Likewise, (4.2) gives $\left\langle E_{j_{1} \ldots j_{n-1}} \sum_{\ell(w)=l} \alpha_{w}\left(\mathfrak{S}_{w}^{q}-\mathcal{Q}_{w}\right)\right\rangle \in \mathbb{Z}_{+}[q]$. Using orthogonality (Theorem 3.9) and (4.3), we rewrite the last statement as

$$
-\sum_{\ell(w)=l} \alpha_{w}\left\langle\left\langle E_{j_{1} \ldots j_{n-1}} \mathcal{Q}_{w}\right\rangle\right\rangle \in \mathbb{Z}_{+}[q] .
$$

Recall that the $\alpha_{w}$ are strictly positive. Comparing (4.4) with (4.5), we conclude that $\left\langle\left\langle E_{j_{1} \ldots j_{n-1}} \mathcal{Q}_{w}\right\rangle=0\right.$, for any $l$, any $w$ of length $l$, and any $j_{1}, \ldots, j_{n-1}$ satisfying (4.3). Therefore $\left\langle\left\langle\mathfrak{S}_{w_{\mathrm{o}} v}^{q} \mathcal{Q}_{w}\right\rangle=0\right.$ for any $v \in S_{n}$ satisfying $\ell(v)<\ell(w)$. Once again using orthogonality, we conclude that the expansion of $\mathcal{Q}_{w}$ in the basis $\left\{\mathfrak{S}_{v}^{q}\right\}$ contains no terms with $\ell(v)<\ell(w)$, meaning that $\mathcal{Q}_{w}=\mathfrak{S}_{w}^{q}$, as desired.

\section{Commuting Difference operators}

Recall that the quotient $\mathbb{Z}[q, x] / I_{n}$ is isomorphic, as a vector space, to the quantum cohomology of the flag manifold (cf. (2.13)). In this section, we construct a family of commuting difference operators acting in $\mathbb{Z}[q, x] / I_{n}$, which will later be shown to correspond to the operators of multiplication by two-dimensional classes 
in the ring $\mathrm{QH}^{*}\left(F l_{n}, \mathbb{Z}\right)$. These operators will be an essential tool in our proof of the orthogonality property, needed for the proof of Theorem 1.2.

Let us identify each polynomial $f \in \mathbb{Z}[q, x]$ with the operator of multiplication by $f$. It will be convenient to denote $q_{i j}=q_{i} q_{i+1} \cdots q_{j-1}$ for $i<j$. Note that $q_{i j} q_{j k}=q_{i k}$. Also let

$$
\partial_{(i j)}=\partial_{t_{i j}}=\partial_{i} \partial_{i+1} \cdots \partial_{j-2} \partial_{j-1} \partial_{j-2} \cdots \partial_{i+1} \partial_{i}
$$

be the divided difference operator corresponding to the transposition $t_{i j}, i<j$.

Define the difference operators $\mathcal{X}_{k}$, for $k=1, \ldots, n$, by

$$
\mathcal{X}_{k}=x_{k}-\sum_{1 \leq i<k} q_{i k} \partial_{(i k)}+\sum_{k<j \leq n} q_{k j} \partial_{(k j)} .
$$

Equivalently, for $\lambda_{1}, \ldots, \lambda_{n} \in \mathbb{Z}[q]$,

$$
\sum_{i} \lambda_{i} \mathcal{X}_{i}=\sum_{i} \lambda_{i} x_{i}+\sum_{1 \leq i<j \leq n}\left(\lambda_{i}-\lambda_{j}\right) q_{i j} \partial_{(i j)} .
$$

Theorem 5.1. The operators $\mathcal{X}_{1}, \ldots, \mathcal{X}_{n}$ commute pairwise.

To prove this result, we will need the following lemma, in which $[$,$] stands for$ the commutator: $[A, B]=A B-B A$.

Lemma 5.2. The following commutation relations hold (recall that we identify the $x_{i}$ with the corresponding multiplication operators):

1. For $a<c$, we have $\left[\partial_{(a c)}, x_{b}\right]=0$ unless $a \leq b \leq c$.

2. For $a<b$, we have $\left[\partial_{(a b)}, x_{a}+x_{a+1}+\cdots+x_{b}\right]=0$.

3. For $a<b$ and $c<d$, we have $\left[\partial_{(a b)}, \partial_{(c d)}\right]=0$ unless $b=c$ or $a=d$.

4. For $a<b<c$, we have $\left[\partial_{(a c)}, x_{b}\right]+\left[\partial_{(a b)}, \partial_{(b c)}\right]=0$.

Proof. 1 . Use (5.1) and the fact that multiplication by $x_{i}$ commutes with $\partial_{j}$ unless $j=i$ or $j=i-1$.

2. Follows from $x_{a}+\cdots+x_{b}$ being a symmetric function of $x_{a}, \ldots, x_{b}$.

3. By Proposition 2.3, $\partial_{v}$ and $\partial_{w}$ commute whenever $v$ and $w$ do. Therefore, $\partial_{(a b)}$ and $\partial_{(c d)}$ commute if the numbers $a, b, c, d$ are all distinct. This proves the claim unless $a=c$ or $b=d$. In these cases, once again using Proposition 2.3, we conclude that $\partial_{(a b)} \partial_{(a d)}=\partial_{(a d)} \partial_{(a b)}=0$ and $\partial_{(a d)} \partial_{(c d)}=\partial_{(c d)} \partial_{(a d)}=0$.

4. From the "Leibniz formula" (2.5) with $w=t_{a c}$, we obtain:

$$
\begin{aligned}
\partial_{(a c)}\left(x_{b} \cdot g\right) & =x_{b} \cdot \partial_{(a c)} g-\partial_{t_{a c} t_{a b}} g+\partial_{t_{a c} t_{b c}} g \\
& =\left(x_{b} \partial_{(a c)}-\partial_{(a b)} \partial_{(b c)}+\partial_{(b c)} \partial_{(a b)}\right) g .
\end{aligned}
$$

Proof of Theorem 5.1. By (5.2) and Lemma 5.2, we have, for $a<b$ :

$$
\begin{aligned}
{\left[\mathcal{X}_{a}, \mathcal{X}_{b}\right]=} & {\left[x_{a},-\sum_{i \leq a} q_{i b} \partial_{(i b)}\right]+\left[\sum_{j \geq b} q_{a j} \partial_{(a j)}, x_{b}\right]+\sum_{i<a} q_{i b}\left[\partial_{(i a)}, \partial_{(a b)}\right] } \\
& +\sum_{j>b} q_{a j}\left[\partial_{(a b)}, \partial_{(b j)}\right]-\sum_{a<i<b} q_{a b}\left[\partial_{(a i)}, \partial_{(i b)}\right] \\
= & -q_{a b}\left[x_{a}, \partial_{(a b)}\right]+q_{a b}\left[\partial_{(a b)}, x_{b}\right]+q_{a b} \sum_{a<i<b}\left[\partial_{(a b)}, x_{i}\right] \\
= & q_{a b}\left[\partial_{(a b)}, x_{a}+x_{a+1}+\cdots+x_{b}\right] \\
= & 0 .
\end{aligned}
$$


For a polynomial $f \in \mathbb{Z}[q, x]$, we write $f(\mathcal{X})$ to denote the operator $f\left(\mathcal{X}_{1}, \ldots, \mathcal{X}_{n}\right)$; this is well defined by Theorem 5.1. Accordingly, $f(\mathcal{X})(g)$ will denote the result of applying $f(\mathcal{X})$ to a polynomial $g$.

Lemma 5.3. For any polynomial $f \in \mathbb{Z}[q, x]$, there exists a unique $F \in \mathbb{Z}[q, x]$ such that $f=F(\mathcal{X})(1)$.

Proof. Let $\operatorname{deg}_{x}$ denote the degree on $\mathbb{Z}[q, x]$ defined by $\operatorname{deg}_{x}\left(x_{i}\right)=1$ and $\operatorname{deg}_{x}\left(q_{j}\right)=$ 0 . We first prove existence by induction on $\operatorname{deg}_{x}(f)=d$. If $d=0$, then $f \in \mathbb{Z}[q]$

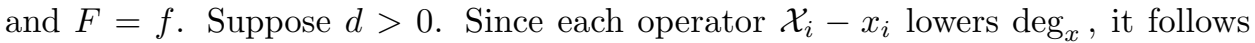
that $f(x)-f(\mathcal{X})(1)$ is a polynomial of degree $<d$. By the induction assumption, this polynomial can be expressed in the form $g(\mathcal{X})(1)$, for some $g \in \mathbb{Z}[q, x]$. Hence $f(x)=(f+g)(\mathcal{X})(1)$. To prove uniqueness, note that whenever $h \neq 0$, one has $\operatorname{deg}_{x}(h(\mathcal{X})(1))=\operatorname{deg}_{x}(h) \geq 0$, implying $h(\mathcal{X})(1) \neq 0$.

As a corollary of the last lemma, commuting operators $\mathcal{X}_{1}, \ldots, \mathcal{X}_{n}$ are algebraically independent over the $\operatorname{ring} \mathbb{Z}[q]$.

By Lemma 5.3 , we have a $\mathbb{Z}[q]$-linear bijection $\psi: \mathbb{Z}[q, x] \rightarrow \mathbb{Z}[q, x]$ given by

$$
\psi: f \mapsto F, \quad f=F(\mathcal{X})(1) .
$$

Our next goal is to find the polynomial $F=\psi(f)$ for the special case $f=e_{i_{1}, \ldots, i_{m}}$.

Proposition 5.4. We have $E_{i}^{k}(\mathcal{X})(g)=e_{i}^{k} g$ for any polynomial $g \in \mathbb{Z}[q, x]$ which is symmetric in the variables $x_{1}, \ldots, x_{k+1}, k<n$, and also in the case $g=1$, $k=n$.

Proof. Induction on $k$. If $k=0$, then $E_{0}^{0}(\mathcal{X})(g)=e_{0}^{0} g=g$. Suppose $k>0$. Then, using the induction hypothesis, Lemma 3.1, (3.1), and (3.5), we obtain:

$$
\begin{aligned}
E_{i}^{k}(\mathcal{X})(g) & =\left(E_{i}^{k-1}(\mathcal{X})+\mathcal{X}_{k} E_{i-1}^{k-1}(\mathcal{X})+q_{k-1} E_{i-2}^{k-2}(\mathcal{X})\right)(g) \\
& =e_{i}^{k-1} g+\mathcal{X}_{k}\left(e_{i-1}^{k-1} g\right)+q_{k-1} e_{i-2}^{k-2} g \\
& =e_{i}^{k-1} g+x_{k} e_{i-1}^{k-1} g-q_{k-1} \partial_{k-1} e_{i-1}^{k-1} g+q_{k-1} e_{i-2}^{k-2} g \\
& =e_{i}^{k-1} g+x_{k} e_{i-1}^{k-1} g \\
& =e_{i}^{k} g .
\end{aligned}
$$

Theorem 5.5. For any $m \leq n$, we have $E_{i_{1} \ldots i_{m}}(\mathcal{X})(1)=e_{i_{1} \ldots i_{m}}$. In particular, $E_{i}^{k}(\mathcal{X})(1)=e_{i}^{k}$ for any $i \leq k \leq n$.

Equivalently, $\psi\left(e_{i_{1} \ldots i_{m}}\right)=\bar{E}_{i_{1} \ldots i_{m}}$ and $\psi\left(e_{i}^{k}\right)=E_{i}^{k}$, in the notation of (5.4).

Proof. Repeatedly using Proposition 5.4, we obtain:

$$
\begin{aligned}
E_{i_{1} \ldots i_{m}}(\mathcal{X})(1) & =E_{i_{1} \ldots i_{m-1}}(\mathcal{X})\left(e_{i_{m}}^{m}\right) \\
& =E_{i_{1} \ldots i_{m-2}}(\mathcal{X})\left(e_{i_{m-1}}^{m-1} e_{i_{m}}^{m}\right)=\cdots=e_{i_{1}}^{1} \cdots e_{i_{m}}^{m} .
\end{aligned}
$$

Corollary 5.6. For any $w \in S_{n}$, we have $\psi\left(\mathfrak{S}_{w}\right)=\mathfrak{S}_{w}^{q}$, i.e., $\mathfrak{S}_{w}^{q}(\mathcal{X})(1)=\mathfrak{S}_{w}$.

Proof. The proof follows from the definition (3.8) of quantum Schubert polynomials. 
Lemma 5.7. The map $\psi$ defined by (5.4) bijectively maps the ideal $I_{n}$ onto $I_{n}^{q}$.

Proof. Every element in $I_{n}$ is of the form $f_{1} e_{n}^{1}+\cdots+f_{n} e_{n}^{n}$, for $f_{1}, \ldots, f_{n} \in \mathbb{Z}[q, x]$. Denote $F_{i}=\psi\left(f_{i}\right)$. Note that each operator $\mathcal{X}_{i}$ commutes with multiplication by any polynomial which is symmetric in $x_{1}, \ldots, x_{n}$ (cf. Proposition 2.4 and (5.2)). Using this observation together with Theorem 5.5, we obtain: $\left(F_{i} E_{i}^{n}\right)(\mathcal{X})(1)=$ $F_{i}(\mathcal{X})\left(e_{i}^{n}\right)=e_{i}^{n} F_{i}(\mathcal{X})(1)=e_{i}^{n} f_{i}$. Hence $\psi\left(f_{1} e_{1}^{n}+\cdots+f_{n} e_{n}^{n}\right)=F_{1} E_{1}^{n}+\cdots+F_{n} E_{n}^{n}$, proving the lemma.

By Lemma 5.7 and Theorem 5.5, the map $\psi$ induces a $\mathbb{Z}[q]$-linear bijection

$$
\mathbb{Z}[q, x] / I_{n} \rightarrow \mathbb{Z}[q, x] / I_{n}^{q},
$$

which sends the cosets of the $e_{i_{1} \ldots i_{m}}$ to the corresponding cosets of $E_{i_{1} \ldots i_{m}}$.

Lemma 5.8. The operators $\mathcal{X}_{1}, \ldots, \mathcal{X}_{n}$ leave the space $I_{n} \subset \mathbb{Z}[q, x]$ invariant.

Proof. We have $\mathcal{X}_{i}\left(f_{1} e_{1}^{n}+\cdots+f_{n} e_{n}^{n}\right)=e_{1}^{n} \mathcal{X}_{i}\left(f_{1}\right)+\cdots+e_{n}^{n} \mathcal{X}_{i}\left(f_{n}\right)$.

Observe that the definition (5.4) of the map $\psi$ implies that $\mathcal{X}_{i}(g)=\psi^{-1}\left(x_{i} \psi(g)\right)$ for any polynomial $g$. In other words, $\psi$ translates the action of $\mathcal{X}_{i}$ into multiplication by $x_{i}$. In view of Lemma $5.8, \mathcal{X}_{i}$ induces an operator in $\mathbb{Z}[q, x] / I_{n}$. This operator corresponds via $\psi$ to multiplication by $x_{i}$ in the $\operatorname{ring} \mathbb{Z}[q, x] / I_{n}^{q}$. It follows that the $\mathcal{X}_{i}$, understood as operators acting in $\mathbb{Z}[q, x] / I_{n}$, satisfy the relations $E_{k}(\mathcal{X})=0$, and thus provide a representation for the ring $\mathrm{QH}\left(F l_{n}, \mathbb{Z}\right)$.

We conclude this section by a useful corollary of Theorem 5.5 and formula (2.7).

Corollary 5.9. Let $F \in \mathbb{Z}[q, x] / I_{n}^{q}$. Then $\langle\langle F\rangle$ is equal to the constant term of $\partial_{w_{\mathrm{o}}}(F(\mathcal{X})(1))$. (The latter is well defined by Lemma 5.7.)

\section{Proof of orthogonality}

In this section we prove the orthogonality property of the quantum Schubert polynomials (Theorem 3.9). To this end, we will need the following lemmas. As before, we identify polynomials with the corresponding multiplication operators.

Lemma 6.1. Let $l \leq k<n$. Then, for any $i_{1}, \ldots, i_{k-1}$,

$$
\partial_{w_{\mathrm{o}}} e_{i_{1}}^{1} \cdots e_{i_{k-1}}^{k-1} \partial_{l} \partial_{l+1} \cdots \partial_{k}=0 \text {. }
$$

Proof. Let us move $\partial_{l}, \ldots, \partial_{k}$ to the left, one by one. By Lemma 3.1 and Proposition 2.4, $\partial_{m}$ commutes with $e_{i}^{a}$ unless $m=a$, whereas

$$
e_{i}^{m} \partial_{m}=\partial_{m} e_{i}\left(x_{1}, \ldots, x_{m-1}, x_{m+1}\right)+e_{i-1}^{m-1} .
$$

If $\partial_{m}$ moves through $e_{i_{m}}^{m}$ (the first term in the right-hand side of (6.2)), then it can be moved all the way to the left, and the corresponding term vanishes since $\partial_{w_{\mathrm{o}}} \partial_{m}=0$. Otherwise $\partial_{m}$ changes $e_{i_{m}}^{m}$ into $e_{i-1}^{m-1}$. The only term remaining in the left-hand side of (6.1) upon moving $\partial_{l}, \ldots, \partial_{k-1}$ will be

$$
\partial_{w_{\mathrm{o}}} e_{i_{1}}^{1} \cdots e_{i_{l-1}}^{l-1} e_{i_{l}-1}^{l-1} \cdots e_{i_{k-1}-1}^{k-2} \partial_{k}=\partial_{w_{\mathrm{o}}} \partial_{k} e_{i_{1}}^{1} \cdots e_{i_{l-1}}^{l-1} e_{i_{l}-1}^{l-1} \cdots e_{i_{k-1}-1}^{k-2}=0 \text {. }
$$

Recall that $L_{n}^{q}$ denotes the $\mathbb{Z}[q]$-span of the monomials $x_{1}^{a_{1}} \cdots x_{n-1}^{a_{n-1}}$ such that $0 \leq a_{i} \leq n-i$ for all $i$. By Proposition 3.4, the polynomials $e_{i_{1} \ldots i_{n-1}}$ form a $\mathbb{Z}[q]$ basis of $L_{n}^{q}$. The following result is proved in exactly the same way as Theorem 5.5. 
Proposition 6.2. For any $f \in L_{n}^{q}$ and any polynomial $g$ which is symmetric in $x_{1}, \ldots, x_{n}$, we have $F(\mathcal{X})(g)=G(\mathcal{X})(f)=f g$, where $F=\psi(f)$ and $G=\psi(g)$, in the notation of (5.4).

Let us fix an integer $k$ such that $1 \leq k \leq n$. It will be convenient to represent the operators $\mathcal{X}_{l}, l \leq k$, in the form $\mathcal{X}_{l}=\widetilde{\mathcal{X}}_{l}+\widetilde{\mathcal{X}}_{l}$, where

$$
\begin{aligned}
& \widetilde{\mathcal{X}}_{l}=x_{l}-\sum_{j<l} q_{j l} \partial_{(j l)}+\sum_{l<j \leq k} q_{l j} \partial_{(l j)}, \\
& \widetilde{\widetilde{\mathcal{X}}}_{l}=\sum_{j>k} q_{l j} \partial_{(l j)} .
\end{aligned}
$$

Let $E_{i}^{k}(\tilde{\mathcal{X}})=E_{i}^{k}\left(\tilde{\mathcal{X}}_{1}, \ldots, \widetilde{\mathcal{X}}_{k}\right)$.

Proposition 6.3. For any polynomial $f \in \mathbb{Z}[q, x]$,

$$
E_{i}^{k}(\tilde{\mathcal{X}})(f)=e_{i}^{k} f
$$

Thus $E_{i}^{k}(\mathcal{X})$ coincides with the operator of multiplication by $e_{i}^{k}$, provided $q_{k}=0$.

Proof. Let us denote $P_{k}=\mathbb{Z}\left[q_{1}, \ldots, q_{k-1}\right]\left[x_{1}, \ldots, x_{k}\right]$. The operator $E_{i}^{k}(\widetilde{\mathcal{X}})$ does not involve divided differences $\partial_{l}$ with $l \geq k$. Therefore it commutes with $x_{l}, l>k$, and it suffices to prove (6.4) for $f \in P_{k}$. (Notice that in this case $E_{i}^{k}(\widetilde{\mathcal{X}})(f) \in P_{k}$. )

Let us expand $f$ in the standard elementary monomials (possibly involving variables $x_{l}$ with $l>k$ ). Let $N$ be greater than the largest value of $M$ such that some $e_{i}^{M}$ enters one of these monomials. Then $f \in L_{N}^{q}$ and, by Proposition 6.2 , we have $E_{i}^{N}(\mathcal{X})(f)=e_{i}^{N} f$. The ideal $J_{k}=\left\langle q_{k}, q_{k+1}, \ldots, x_{k+1}, x_{k+2}, \ldots\right\rangle$ is an invariant subspace for all the $\mathcal{X}_{i}$ and for the operators of multiplication by $x_{i}$. Hence $\mathcal{X}_{i}$ and $x_{i}$ act on the quotient space, which is isomorphic to $P_{k}$. The corresponding actions of $E_{i}^{N}(\mathcal{X})$ and $e_{i}^{N}$ on $P_{k}$ coincide with $E_{i}^{k}(\widetilde{\mathcal{X}})$ and $e_{i}^{k}$, respectively. This implies $E_{i}^{k}(\widetilde{\mathcal{X}})(f)=e_{i}^{k} f$, as desired.

Lemma 6.4. For any $k<n$ and any $f$,

$$
\partial_{w_{\mathrm{o}}} e_{i_{1} \ldots i_{k-1}}\left(E_{i_{k}}^{k}(\mathcal{X})-e_{i_{k}}^{k}\right) f=0 \text {. }
$$

Proof. Let us write $E_{i_{k}}^{k}(\mathcal{X})$ as a polynomial in the $\mathcal{X}_{l}, l \leq k$, substitute $\mathcal{X}_{l}=\widetilde{\mathcal{X}}_{l}+\widetilde{\widetilde{\mathcal{X}}}_{l}$ everywhere, and expand. The terms which only involve the truncated operators $\widetilde{\mathcal{X}}_{l}$ will combine into the operator $E_{i_{k}}^{k}(\widetilde{\mathcal{X}})$. By Proposition $6.3,\left(E_{i_{k}}^{k}(\widetilde{\mathcal{X}})-e_{i_{k}}^{k}\right) f=0$. To prove Lemma 6.4 , it therefore suffices to show that any composition of operators of the form

$$
\partial_{w_{\mathrm{o}}} e_{i_{1} \ldots i_{k-1}} \widetilde{\mathcal{X}}_{l_{1}} \ldots \widetilde{\mathcal{X}}_{l_{j}} \partial_{(l m)}
$$

with $1 \leq l_{1}<l_{2}<\cdots<l_{j}<l \leq k<m$, vanishes. In view of the definition of $\partial_{(l m)}$, this claim will follow if we prove that

$$
\partial_{w_{\mathrm{o}}} e_{i_{1} \ldots i_{k-1}} \tilde{\mathcal{X}}_{l_{1}} \ldots \widetilde{\mathcal{X}}_{l_{j}} \partial_{l} \cdots \partial_{k}=0
$$

for $1 \leq l_{1}<l_{2}<\cdots<l_{j}<l \leq k$. We will prove (6.7) by induction on $j$. If $j=0$, then it coincides with Lemma 6.1. For $j>0$, the only term in the expression (6.3) for $\widetilde{\mathcal{X}}_{l_{j}}$ that neither commutes with $\left(\partial_{l} \cdots \partial_{k}\right)$ nor vanishes after composition with $\left(\partial_{l} \cdots \partial_{k}\right)$ is $q_{l_{j} l} \partial_{\left(l_{j} l\right)}$. We then note that

$$
\partial_{\left(l_{j} l\right)}\left(\partial_{l} \cdots \partial_{k}\right)=\partial_{l_{j}} \cdots \partial_{l-2} \partial_{l-1} \partial_{l-2} \cdots \partial_{l_{j}}\left(\partial_{l} \cdots \partial_{k}\right)=\left(\partial_{l_{j}} \cdots \partial_{k}\right) \partial_{l-2} \cdots \partial_{l_{j}} .
$$


Using the induction assumption, we conclude that the left-hand side of (6.7) equals

$$
q_{l_{j} l}\left(\partial_{w_{\mathrm{o}}} e_{i_{1} \ldots i_{k-1}} \widetilde{\mathcal{X}}_{l_{1}} \cdots \widetilde{\mathcal{X}}_{l_{j-1}} \partial_{l_{j}} \partial_{l_{j}+1} \cdots \partial_{k}\right) \partial_{l-2} \cdots \partial_{l_{j}} .
$$

The parenthesized factor is an expression similar to the left-hand side of (6.7) with $j$ decreased by 1 and $l$ replaced by $l_{j}$. By the induction assumption, it vanishes, and Lemma 6.4 is proved.

We will now complete the proof of Theorem 3.9. Let us first note that the only nontrivial case is $\ell(u)+\ell(v)>\ell\left(w_{\mathrm{o}}\right)$. Indeed, if $\operatorname{deg}\left(\mathfrak{S}_{u}^{q} \mathfrak{S}_{v}^{q}\right)=\ell(u)+\ell(v)<\ell\left(w_{\mathrm{o}}\right)$, then the monomial $x^{\delta}$ cannot appear in the expansion of $\mathfrak{S}_{u}^{q} \mathfrak{S}_{v}^{q}$. In the case $\ell(u)+\ell(v)=\ell\left(w_{\mathrm{o}}\right)$, the only terms which can contribute to $\left\langle\left\langle\mathfrak{S}_{u}^{q} \mathfrak{S}_{v}^{q}\right\rangle\right.$ are those not involving the $q_{i}$, and (3.9) follows from its classical counterpart (2.9).

Since our quantum Schubert polynomials are linear combinations of quantum standard elementary monomials of the same degree, it remains to show that

$$
\left\langle\left\langle E_{i_{1} \ldots i_{n-1}} E_{j_{1} \ldots j_{n-1}}\right\rangle=0\right.
$$

whenever

$$
i_{1}+\cdots+i_{n-1}+j_{1}+\cdots+j_{n-1}>\ell\left(w_{\mathrm{o}}\right)=n(n-1) / 2 .
$$

In view of Theorem 5.5, $E_{i_{1} \ldots i_{n-1}}(\mathcal{X}) E_{j_{1} \ldots j_{n-1}}(\mathcal{X})(1)=E_{i_{1} \ldots i_{n-1}}(\mathcal{X})\left(e_{j_{1} \ldots j_{n-1}}\right)$. By Corollary 5.9 , formula (6.8) is equivalent to

$$
\left(\partial_{w_{\mathrm{o}}}\left(E_{i_{1} \ldots i_{n-1}}(\mathcal{X})\left(e_{j_{1} \ldots j_{n-1}}\right)\right)\right)(0, \ldots, 0)=0 .
$$

Suppressing $(\mathcal{X})$ to avoid cumbersome notation, let us write

$$
E_{i_{1} \ldots i_{n-1}}=E_{i_{1}}^{1} \cdots E_{i_{n-1}}^{n-1}=\left(e_{i_{1}}^{1}+\left(E_{i_{1}}^{1}-e_{i_{1}}^{1}\right)\right) \cdots\left(e_{i_{n-1}}^{n-1}+\left(E_{i_{n-1}}^{n-1}-e_{i_{n-1}}^{n-1}\right)\right)
$$

and prove that for each term $T$ in the expansion of this product we have

$$
\left(\partial_{w_{\mathrm{o}}} T\left(e_{j_{1} \ldots j_{n-1}}\right)\right)(0, \ldots, 0)=0 .
$$

If $T=e_{i_{1}}^{1} \cdots e_{i_{n-1}}^{n-1}$, then (6.11) follows from (6.9). Any other $T$ is of the form

$$
T=e_{i_{1}}^{1} \cdots e_{i_{k-1}}^{k-1}\left(E_{i_{k}}^{k}-e_{i_{k}}^{k}\right) T^{\prime}
$$

for some $k \leq n-1$, and (6.11) follows from Lemma 6.4 with $g=T^{\prime}\left(e_{j_{1} \ldots j_{n-1}}\right)$. This completes the proof of Theorem 3.9.

Define a bilinear form $(,$,$) in \mathbb{Z}[q, x] / I_{n}^{q}$ by setting

$$
\left(F, \mathfrak{S}_{w}^{q}\right)=\left\langle\left\langle F \mathfrak{S}_{w_{\mathrm{o}} w}^{q}\right\rangle .\right.
$$

The following result is a reformulation of Theorem 3.9.

Theorem 6.5. With respect to the scalar product $(6.12)$ in $\mathbb{Z}[q, x] / I_{n}^{q}$, the quantum Schubert polynomials $\mathfrak{S}_{w}^{q}, w \in S_{n}$, form an orthonormal basis. 


\section{QuANTUM MONK's FORMUla}

In this section, we prove the quantum Monk's formula (Theorem 1.3). Let us first reformulate this formula in the language of quantum Schubert polynomials.

Theorem 7.1 (Quantum Monk's formula). For $w \in S_{n}$ and $1 \leq r<n$,

$$
\mathfrak{S}_{s_{r}}^{q} \mathfrak{S}_{w}^{q}=\left(x_{1}+\cdots+x_{r}\right) \mathfrak{S}_{w}^{q}=\sum \mathfrak{S}_{w t_{a b}}^{q}+\sum q_{c d} \mathfrak{S}_{w t_{c d}}^{q},
$$

where the first sum is over all transpositions $t_{a b}$ such that $a \leq r<b$ and $\ell\left(w t_{a b}\right)=$ $\ell(w)+1$, and the second sum is over all transpositions $t_{c d}$ such that $c \leq r<d$ and $\ell\left(w t_{c d}\right)=\ell(w)-\ell\left(t_{c d}\right)=\ell(w)-2(d-c)+1$.

More generally, for any linear form $f=\sum \lambda_{i} x_{i}$, one has

$$
f \mathfrak{S}_{w}^{q}=\sum\left(\lambda_{a}-\lambda_{b}\right) \mathfrak{S}_{w t_{a b}}^{q}+\sum\left(\lambda_{c}-\lambda_{d}\right) q_{c d} \mathfrak{S}_{w t_{c d}}^{q},
$$

where the sums are over $a<b$ and $c<d$ such that $\ell\left(w t_{a b}\right)=\ell(w)+1$ and $\ell\left(w t_{c d}\right)=\ell(w)-\ell\left(t_{c d}\right)$, respectively.

Proof. By the classical Monk's formula (Theorem 2.8), the definition of $\mathfrak{S}_{w}^{q}$, and Theorem 5.5, the equation (7.2) is equivalent to

$$
f(\mathcal{X})\left(\mathfrak{S}_{w}\right)=f \cdot \mathfrak{S}_{w}+\sum\left(\lambda_{c}-\lambda_{d}\right) q_{c d} \mathfrak{S}_{w t_{c d}}
$$

summed over all $c<d$ such that $\ell\left(w t_{c d}\right)=\ell(w)-\ell\left(t_{c d}\right)$. The latter follows from Corollary 2.7.

Notice that formulas (7.1) and (7.2) hold on the level of polynomials, not just for cosets (classes), as in Theorem 1.3.

\section{QUANTIZATION MAP}

At this point, let us review our main results, now that all of them are proved.

In view of Theorems 1.1, 5.5 (cf. also (5.5)) and 7.1, we now have four different descriptions of the quantization map $\mathbb{Z}[q, x] / I_{n} \rightarrow \mathbb{Z}[q, x] / I_{n}^{q}$ defined by the commutative diagram (1.5). Geometrically, this is the map that sends a coset that corresponds to a given class in the ordinary cohomology of $F l_{n}$ to the coset corresponding to the same class as an element of the quantum cohomology ring. Algebraically (or combinatorially), this map is defined by its action on standard elementary monomials:

$$
e_{i_{1} \ldots i_{n-1}} \longmapsto E_{i_{1} \ldots i_{n-1}} \text {. }
$$

The quantization map can also be defined in terms of the operators $\mathcal{X}_{k}$ given by (5.2). Namely, the quantization image $F$ of $f$ is uniquely determined by

$$
f=\left(F\left(\mathcal{X}_{1}, \ldots, \mathcal{X}_{n}\right)\right)(1)
$$

Yet another description can be obtained from the quantum Monk's formula. It is not hard to see that this formula can be used to recursively obtain the quantum Schubert polynomials, starting with $\mathfrak{S}_{1}^{q}=1$. The quantization map is then defined by

$$
\mathfrak{S}_{w} \longmapsto \mathfrak{S}_{w}^{q}
$$




\section{Axiomatic CHARACTERIZATion}

In Section 4, we have actually proved the following characterization theorem.

Theorem 9.1. Let $\left\{\mathcal{Q}_{w}\right\}_{w \in S_{n}}$ be a family of elements of $\mathbb{Z}[q, x] / I_{n}^{q}$ satisfying Properties 4.1-4.4. Then the $\mathcal{Q}_{w}$ are the cosets of the quantum Schubert polynomials $\mathfrak{S}_{w}^{q}$.

It is natural to ask exactly which properties are essential to uniquely determine the elements $\mathcal{Q}_{w}$. In particular, does one really need Property 4.4 , which is the only property in Theorem 9.1 that does not immediately follow from the definition of the quantum cohomology of $F l_{n}$ ?

Proposition 9.2. In the case of $S_{3}$ (or $\mathrm{Fl}_{3}$ ), Properties 4.1-4.3 uniquely determine the $\mathcal{Q}_{w}$ (which hence coincide with the cosets of the $\mathfrak{S}_{w}^{q}$ ).

Proof. We certainly know that $\mathcal{Q}_{1}=1, \mathcal{Q}_{s_{1}}=x_{1}$, and $\mathcal{Q}_{s_{2}}=x_{1}+x_{2}$. Assume that $\mathcal{Q}_{s_{1} s_{2}}=\mathfrak{S}_{s_{1} s_{2}}^{q}+a$ and $\mathcal{Q}_{s_{2} s_{1}}=\mathfrak{S}_{s_{2} s_{1}}^{q}+b$, where $a$ and $b$ are, by Properties 4.1 and 4.2, some linear combinations of $q_{1}$ and $q_{2}$. Then direct computations (with or without a computer) give $\left\langle\left\langle\mathcal{Q}_{s_{1} s_{2}}\left(\mathcal{Q}_{s_{1}}\right)^{2} \mathcal{Q}_{s_{2}}\right\rangle=a\right.$ and $\left\langle\left\langle\mathcal{Q}_{s_{2} s_{1}}\left(\mathcal{Q}_{s_{2}}\right)^{2} \mathcal{Q}_{s_{1}}\right\rangle=b\right.$, implying that both $a$ and $b$ are nonnegative linear combinations of $q_{1}$ and $q_{2}$ (we will simply write $a \geq 0$ and $b \geq 0$ ). On the other hand,

$$
\mathcal{Q}_{s_{1}} \mathcal{Q}_{s_{2}}=x_{1}\left(x_{1}+x_{2}\right)=\mathfrak{S}_{s_{1} s_{2}}^{q}+\mathfrak{S}_{s_{2} s_{1}}^{q}=\mathcal{Q}_{s_{1} s_{2}}+\mathcal{Q}_{s_{2} s_{1}}+(-a-b) .
$$

Hence $-a-b \geq 0$ and therefore $a=b=0$, as desired. The remaining case of $\mathcal{Q}_{w_{\mathrm{o}}}$ is treated in analogous fashion. Assume $\mathcal{Q}_{w_{\mathrm{o}}}=\mathfrak{S}_{w_{\mathrm{o}}}^{q}+c \mathfrak{S}_{s_{1}}^{q}+d \mathfrak{S}_{s_{2}}^{q}$. Then $\left\langle\left\langle\mathcal{Q}_{w_{\mathrm{o}}} \mathcal{Q}_{s_{1} s_{2}}\right\rangle=\left\langle\left\langle\mathcal{Q}_{w_{\mathrm{o}}} \mathfrak{S}_{s_{1} s_{2}}^{q}\right\rangle=c\right.\right.$ and $\left\langle\left\langle\mathcal{Q}_{w_{\mathrm{o}}} \mathcal{Q}_{s_{2} s_{1}}\right\rangle=\left\langle\left\langle\mathcal{Q}_{w_{\mathrm{o}}} \mathfrak{S}_{s_{2} s_{1}}^{q}\right\rangle=d\right.\right.$, implying $c \geq 0$ and $d \geq 0$. On the other hand, $\mathcal{Q}_{s_{1}} \mathcal{Q}_{s_{1} s_{2}}=\mathfrak{S}_{w_{\mathrm{o}}}^{q}=\mathcal{Q}_{w_{\mathrm{o}}}-c \mathcal{Q}_{s_{1}}-d \mathcal{Q}_{s_{2}}$. Hence $-c \geq 0$ and $-d \geq 0$, and therefore $c=d=0$, as desired.

Similar but more involved considerations allowed us to verify, with the help of a computer, that the result analogous to Proposition 9.2 holds for $n=4$. It is tempting to conjecture that this holds for every $n$. This would be a very nice result (perhaps too nice to be true), since it would mean that it suffices to use the basic properties of quantum cohomology to uniquely determine the polynomials $\mathfrak{S}_{w}^{q}$.

Conjecture 9.3 (Strong version). The quantum Schubert polynomials are uniquely defined by Properties 4.1-4.3. In other words, they are the only elements of $\mathbb{Z}[q, x] / I_{n}^{q}$ which are homogeneous with respect to the grading $\operatorname{deg}\left(x_{i}\right)=1, \operatorname{deg}\left(q_{j}\right)=$ 2 , specialize to the ordinary Schubert polynomials in the classical limit $q_{1}=\cdots=$ $q_{n-1}=0$, and whose structure constants are polynomials in the $q_{i}$ with nonnegative integer coefficients.

A weaker conjecture (cf. Conjecture 9.7 below) would extend the defining set of axioms by additional properties of the elements $\mathcal{Q}_{w}$, which can be directly derived from the their geometric definition. The first of these extra properties is orthogonality (cf. Theorem 3.9).

Property 9.4. For $u, v \in S_{n}$,

$$
\left\langle\left\langle\mathcal{Q}_{u} \mathcal{Q}_{v}\right\rangle\right\rangle= \begin{cases}1 & \text { if } v=w_{\mathrm{o}} u \\ 0 & \text { otherwise. }\end{cases}
$$


Proof. By definition, $\left\langle\left\langle\mathcal{Q}_{u} \mathcal{Q}_{v}\right\rangle\right\rangle$ is the coefficient of $\mathcal{Q}_{w_{\mathrm{o}}}$ in $\mathcal{Q}_{u} \mathcal{Q}_{v}$, which is equal to the coefficient of the Schubert cycle $\sigma_{w_{\circ}}$ in the quantum product $\sigma_{u} * \sigma_{v}$. In view of (2.14) and the $S_{3}$-symmetry of the Gromov-Witten invariants, this is the same as the coefficient of $\sigma_{w_{\mathrm{o}} u}$ in $\sigma_{v} * \sigma_{1}=\sigma_{v}$, which obviously equals the right-hand side of (9.1).

The $S_{2}$-symmetry of the type $A_{n-1}$ Dynkin diagram has as a consequence the invariance of the quantum (and, in particular, the ordinary) cohomology of the flag manifold with respect to the involutive ring automorphism $\omega: \mathrm{QH}^{*}\left(F l_{n}\right) \rightarrow$ $\mathrm{QH}^{*}\left(F l_{n}\right)$ defined by $\omega\left(\sigma_{s_{k}}\right)=\sigma_{s_{n-k}}$ and $\omega\left(q_{k}\right)=q_{n-k}$ for $k=1,2, \ldots, n-1$. Since $\mathcal{Q}_{s_{k}}=\mathfrak{S}_{s_{k}}^{q}=x_{1}+\cdots+x_{k}$, and $x_{1}+\cdots+x_{n} \in I_{n}^{q}$, we can alternatively define $\omega$ as the ring automorphism of $\mathbb{Z}[q, x] / I_{n}^{q}$ given by

$$
\omega\left(x_{k}\right)=-x_{n+1-k}, \quad \omega\left(q_{k}\right)=q_{n-k} .
$$

Property 9.5. For any $w \in S_{n}$, one has $\omega\left(\mathcal{Q}_{w}\right)=\mathcal{Q}_{w_{\mathrm{o}} w w_{\mathrm{o}}}$.

The involution $\omega$ is studied in Section 11.1. In particular, Property 9.5 can be proved combinatorially (cf. Corollary 11.6).

Property 9.6. If $u$ and $v$ belong to the parabolic subgroups generated by $s_{1}, \ldots, s_{k}$ and $s_{k+1}, \ldots, s_{n-1}$, respectively, for some $k$, then $\mathcal{Q}_{u v}=\mathcal{Q}_{u} \mathcal{Q}_{v}$.

Conjecture 9.7 (Weak version). The quantum Schubert polynomials are uniquely defined by Properties 4.1-4.3 and 9.4-9.6.

\section{Stability}

The quantum Schubert polynomials have an important stability property that justifies their choice as specific polynomial representatives of the corresponding cosets modulo the ideal $I_{n}^{q}$. Consider the natural embeddings of the symmetric groups $S_{1} \subset S_{2} \subset S_{3} \subset \cdots$; viz., $S_{n}$ permutes the set $\{1, \ldots, n\}$.

Theorem 10.1 (Stability). Let $w \in S_{n}$. The quantum Schubert polynomial $\mathfrak{S}_{w}^{q}$ is the unique polynomial in $\mathbb{Z}\left[q_{1}, \ldots, q_{n-1}\right]\left[x_{1}, \ldots, x_{n}\right]$ which has the following property: for every $N \geq n$, the polynomial $\mathfrak{S}_{w}^{q}$ represents the Schubert class $\sigma_{w}$, in the quantum cohomology ring $\mathrm{QH}^{*}\left(F l_{N}\right) \cong \mathbb{Z}\left[q_{1}, \ldots, q_{N-1}\right]\left[x_{1}, \ldots, x_{N}\right] / I_{N}^{q}$.

Proof. It is immediate from our definition that the quantum Schubert polynomial $\mathfrak{S}_{w}^{q}$, for $w \in S_{n}$, does not change if $w$ is regarded as an element of $S_{N}$, for any $N>n$. Hence the property in question follows from Theorem 1.2. To prove uniqueness, it suffices to show that if a polynomial $f \in \mathbb{Z}\left[q_{1}, \ldots, q_{n-1}\right]\left[x_{1}, \ldots, x_{n}\right]$ vanishes modulo the ideal $I_{N}^{q}$, for all $N>n$, then it vanishes identically. Indeed, $f$ belongs to the $\mathbb{Z}\left[q_{1}, \ldots, q_{N-1}\right]$-span of the monomials $x_{1}^{a_{1}} \cdots x_{N-1}^{a_{N-1}}, 0 \leq a_{k} \leq N-k$, for $N \geq n+\operatorname{deg}_{x}(f)$. Therefore, by Proposition 3.6, $f=0$ if $f \in I_{N}^{q}$.

Several properties of the quantum Schubert polynomials $\mathfrak{S}_{w}^{q}$ are peculiar to the particular choice of coset representatives that we made. The proofs of the following statements are left to the reader:

- the quantum Schubert polynomials $\mathfrak{S}_{w}^{q}$, for $w \in S_{\infty}$, where $S_{\infty}=\bigcup S_{n}$, form a $\mathbb{Z}\left[q_{1}, q_{2}, \ldots\right]$-linear basis of the polynomial ring $\mathbb{Z}\left[q_{1}, q_{2}, \ldots\right]\left[x_{1}, x_{2}, \ldots\right]$;

- to multiply $\mathfrak{S}_{u}^{q}$ and $\mathfrak{S}_{v}^{q}$ in the quotient ring $\mathbb{Z}[q, x] / I_{n}^{q}$ (which is equivalent to computing the quantum product $\sigma_{u} * \sigma_{v}$ of the corresponding Schubert classes), expand $\mathfrak{S}_{u}^{q} \mathfrak{S}_{v}^{q}$ in the basis $\left\{\mathfrak{S}_{w}^{q}\right\}$ of the ring $\mathbb{Z}\left[q_{1}, q_{2}, \ldots\right]\left[x_{1}, x_{2}, \ldots\right]$ and drop all terms containing $\mathfrak{S}_{w}^{q}$ with $w$ not in $S_{n}$; 
- $\mathfrak{S}_{w}^{q}$ does not involve $q_{n-1}$;

- if $w \in S_{n}$ but $w \notin S_{n-1}$, then $\mathfrak{S}_{w}^{q} \in I_{n-1}^{q}$.

\section{Miscellaneous}

11.1. Quantum complete homogeneous polynomials. Let $h_{l}^{k}$ denote the sum of all monomials of degree $l$ in the variables $x_{1}, \ldots, x_{k}$ (the complete homogeneous symmetric polynomial). The following result is well known.

Lemma 11.1. For $k+l>n$, one has $h_{l}^{k} \in I_{n}$.

Proof. The statement follows from the formula $h_{l}^{k}=\operatorname{det}\left(e_{j-i+1}^{k+l-i}\right)_{i, j=1}^{l}$ since all elements in the first row of this determinant belong to $I_{n}$.

The proofs of the remaining results in Section 11 are fairly straightforward, and are omitted for the sake of brevity.

Theorem 11.2. The quantization map sends the coset of a complete homogeneous polynomial $h_{l}^{k}$ to $H_{l}^{k}=\operatorname{det}\left(E_{j-i+1}^{k+l-i}\right)_{i, j=1}^{l}$. More generally, the quantization of $h_{i_{1} \ldots i_{n-1}}=h_{i_{1}}^{1} \cdots h_{i_{n-1}}^{n-1}$, where $i_{k} \leq n-k$ for $k=1, \ldots, n-1$, is

$$
H_{i_{1} \ldots i_{n-1}}=H_{i_{1}}^{1} \cdots H_{i_{n-1}}^{n-1} .
$$

Observe that if the condition $i_{k} \leq n-k$ is not satisfied for at least one value of $k$, then $h_{i_{1} \ldots i_{n-1}} \in I_{n}$, by Lemma 11.1 .

The quantum complete homogeneous polynomials $H_{l}^{k}$ will play a role in Section 12.1 as elements of a Gröbner basis for the ideal $I_{n}^{q}$. These polynomials can be given a direct combinatorial interpretation in terms of families of nonintersecting paths in a certain oriented graph.

11.2. Involution $\omega$. Let $\omega$ be the involutive automorphism of the polynomial ring $\mathbb{Z}[q, x]$ defined by $\omega\left(x_{k}\right)=-x_{n+1-k}$ and $\omega\left(q_{k}\right)=q_{n-k}$, for $k=1, \ldots, n$ (cf. $(9.2)$ ).

Lemma 11.3. Both $I_{n}$ and $I_{n}^{q}$ are invariant subspaces for the involution $\omega$.

This shows that $\omega$ has well-defined actions on both $\mathbb{Z}[q, x] / I_{n}$ and $\mathbb{Z}[q, x] / I_{n}^{q}$.

Proposition 11.4 (cf. [21]). In $\mathbb{Z}[q, x] / I_{n}$, the following rules for computing $\omega$ images hold:

$$
\omega\left(e_{i_{1} \ldots i_{n-1}}\right)=h_{i_{n-1} \ldots i_{1}} ; \quad \omega\left(h_{i_{1} \ldots i_{n-1}}\right)=e_{i_{n-1} \ldots i_{1}} ; \quad \omega\left(\mathfrak{S}_{w}\right)=\mathfrak{S}_{w_{\mathrm{o}} w w_{\mathrm{o}}} .
$$

Proposition 11.5. Involution $\omega$ commutes with the quantization map.

Corollary 11.6. In $\mathbb{Z}[q, x] / I_{n}^{q}$, the following rules for computing $\omega$-images hold:

$$
\omega\left(E_{i_{1} \ldots i_{n-1}}\right)=H_{i_{n-1} \ldots i_{1}} ; \quad \omega\left(H_{i_{1} \ldots i_{n-1}}\right)=E_{i_{n-1} \ldots i_{1}} ; \quad \omega\left(\mathfrak{S}_{w}^{q}\right)=\mathfrak{S}_{w_{\mathrm{o}} w w_{\mathrm{o}}}^{q} .
$$

11.3. Quantization of square-free monomials. The combinatorial construction of Section 3.2 can be used to describe the image of any square-free monomial $x_{a}=x_{a_{1}} x_{a_{2}} \cdots$ under the quantization map. Consider the graph whose vertices are the $a_{i}$ and whose edges connect $a_{i}$ and $a_{j}$ if $\left|a_{i}-a_{j}\right|=1$. Assign weight $x_{a_{i}}$ to the vertex $a_{i}$ and weight $q_{a_{i}}$ to the edge $\left(a_{i}, a_{i}+1\right)$. Then every matching in this graph (a collection of vertex-disjoint edges) acquires a weight equal to the product of weights of its edges multiplied by the weights of remaining vertices. The sum of 
these weights, for all matchings, is the quantization of the monomial $x_{a}$. A similar rule for computing the inverse ("dequantization") image $\mathcal{X}_{a_{1}} \mathcal{X}_{a_{2}} \cdots(1)$ of a squarefree monomial $x_{a}$ can be obtained using Möbius inversion. The only difference from the quantization rule is in replacing each $q_{i}$ by $-q_{i}$.

\section{Explicit COMPUTATION}

12.1. Gröbner bases. Recall that $L_{n}^{q}$ denotes the space, complementary to the ideal $I_{n}^{q}$, which is spanned (over $\mathbb{Z}[q]$ ) by the monomials $x_{1}^{a_{1}} \cdots x_{n-1}^{a_{n-1}}$ satisfying $0 \leq a_{k} \leq n-k$. Another basis of $L_{n}^{q}$ is formed by the quantum Schubert polynomials $\mathfrak{S}_{w}^{q}$, for $w \in S_{n}$ (see Proposition 3.7). The problem of finding the unique representative in $L_{n}^{q}$ of a given coset modulo $I_{n}^{q}$ can be solved using Gröbner bases techniques. We refer the reader to [29, Chapter 1], which contains all definitions and facts that we will need from the theory of Gröbner bases.

Let us use the degree lexicographic order, induced from $x_{1} \prec x_{2} \prec \cdots \prec x_{n}$, on the set of all monomials $x_{1}^{a_{1}} \cdots x_{n}^{a_{n}}$. More precisely, we first order the monomials by the total degree $a_{1}+\cdots+a_{n}$, and then break the ties using the lexicographic order on the sequences $\left(a_{n}, \ldots, a_{1}\right)$. This allows us to introduce the normal form of any polynomial with respect to the ideal $I_{n}^{q}$ and the monomial order specified above. This normal form can be found by means of a reduction procedure versus the corresponding reduced Gröbner basis $G$ of $I_{n}^{q}$. The following direct description of this Gröbner basis and the space of normal forms can be viewed as the quantum analogue of [29, Theorem 1.2.7].

Proposition 12.1. The vector space $L_{n}^{q}$ is the space of normal forms for the ideal $I_{n}^{q} \subset \mathbb{Z}[q, x]$ with respect to the degree lexicographic monomial order defined above.

The corresponding reduced Gröbner basis $G$ consists of the quantum complete homogeneous polynomials $H_{k}^{n+1-k}, k=1, \ldots, n$ (see Theorem 11.2).

Proof. We already know that each coset modulo $I_{n}^{q}$ contains a unique representative from $L_{n}^{q}$. Let us show that any monomial outside $L_{n}^{q}$ is congruent modulo $I_{n}^{q}$ to a sum of smaller monomials. Assume $x^{a}=x_{1}^{a_{1}} \cdots x_{n}^{a_{n}} \notin L_{n}^{q}$, which means that $a_{k}>n-k$ for some $k$. Then, by Lemma 11.1, $h_{a_{k}}^{k} \in I_{n}$, implying $H_{a_{k}}^{k} \in I_{n}^{q}$. Note that $x_{k}^{a_{k}}$ is the largest monomial in the expansion of $H_{a_{k}}^{k}$. Hence $x_{k}^{a_{k}}$ can be written, modulo $I_{n}^{q}$, as a linear combination of smaller monomials. Multiplying by all the $x_{i}^{a_{i}}$ with $i \neq k$, we obtain the desired expansion of $x^{a}$ into smaller terms.

The description of the Gröbner basis $G$ is then derived from the following two facts: (i) the monomials outside $L_{n}^{q}$ are exactly those divisible by some monomial of the form $x_{n+1-k}^{k}$, and (ii) a leading monomial of $H_{k}^{n+1-k}$ is $x_{n+1-k}^{k}$.

To illustrate, for $n=3$ the space of normal forms is the $\mathbb{Z}\left[q_{1}, q_{2}\right]$-span of the monomials $1, x_{1}, x_{1}^{2}, x_{2}, x_{1} x_{2}, x_{1}^{2} x_{2}$. The reduced minimal Gröbner basis for $I_{3}^{q}$ consists of

$$
\begin{aligned}
& H_{1}^{3}=x_{1}+x_{2}+x_{3}, \\
& H_{2}^{2}=x_{1}^{2}+x_{1} x_{2}+x_{2}^{2}-q_{1}-q_{2},
\end{aligned}
$$

and

$$
H_{3}^{1}=x_{1}^{3}-2 x_{1} q_{1}-x_{2} q_{1}
$$


12.2. Computing the Gromov-Witten invariants. Since the quantum Schubert polynomials $\mathfrak{S}_{w}^{q}$ represent Schubert classes, the structure constants of the ring $\mathbb{Z}[q, x] / I_{n}^{q}$ with respect to the basis $\left\{\mathfrak{S}_{w}^{q}\right\}$ are the generating functions for the Gromov-Witten invariants $\left\langle\sigma_{u}, \sigma_{v}, \sigma_{w}\right\rangle_{d}$ of the flag manifold (cf. (2.14)). Actually, (2.14) generalizes to

$$
\sigma_{w_{1}} * \cdots * \sigma_{w_{k}}=\sum_{w \in S_{n}} \sum_{d} q^{d}\left\langle\sigma_{w}, \sigma_{w_{1}}, \ldots, \sigma_{w_{k}}\right\rangle_{d} \sigma_{w_{\mathrm{o}} w}
$$

(see, e.g., [7]). This formula allows us to compute $k$-point Gromov-Witten invariants for an arbitrary $k$.

Corollary 12.2. For any $w_{1}, \ldots, w_{k} \in S_{n}$,

$$
\left\langle\left\langle\mathfrak{S}_{w_{1}}^{q} \ldots \mathfrak{S}_{w_{k}}^{q}\right\rangle\right\rangle=\sum_{d} q^{d}\left\langle\sigma_{w_{1}}, \ldots, \sigma_{w_{k}}\right\rangle_{d} .
$$

Proof. This is a consequence of (12.1) and the orthogonality property (3.9).

Combining (12.2) with Corollary 5.9, we obtain the following formula for the generating function of the Gromov-Witten invariants.

Corollary 12.3. For any $w_{1}, \ldots, w_{k} \in S_{n}$,

$$
\sum_{d} q^{d}\left\langle\sigma_{w_{1}}, \ldots, \sigma_{w_{k}}\right\rangle_{d}=\left\langle\left(\prod_{j} \mathfrak{S}_{w_{j}}^{q}\left(\mathcal{X}_{1}, \ldots, \mathcal{X}_{n-1}\right)\right)(1)\right\rangle
$$

where $\langle f\rangle$ denotes the constant term of $\partial_{w_{\mathrm{o}}}(f)(c f .(2.7)$ and $(2.8))$.

An efficient alternative to the last formula is provided by a method described below, which is based on the Gröbner bases techniques developed in Section 12.1.

Corollary 12.4. The expansion of an element $F \in \mathbb{Z}[q, x] / I_{n}^{q}$ in the basis of cosets of quantum Schubert polynomials $\mathfrak{S}_{w}^{q}, w \in S_{n}$, is given by $F=\sum c_{w} \mathfrak{S}_{w}^{q} \bmod I_{n}^{q}$, where each $c_{w}$ is the coefficient of the staircase monomial $x^{\delta}=x_{1}^{n-1} x_{2}^{n-2} \cdots x_{n-1}$ in the normal form of the polynomial $F \mathfrak{S}_{w_{\mathrm{o}} w}^{q}$ with respect to the degree lexicographic monomial order described in Section 12.1.

Proof. In view of Theorem 3.9, $c_{w}$ is equal to the coefficient of $\mathfrak{S}_{w_{\mathrm{o}}}^{q}$ in the expansion of $F \mathfrak{S}_{w_{\mathrm{o}} w}^{q}$. By Proposition 12.1, the normal form of $F \mathfrak{S}_{w_{\mathrm{o}} w}^{q}$ lies in $L_{n}^{q}$, and the coefficient of $\mathfrak{S}_{w_{\mathrm{o}}}^{q}$ in its expansion in the basis of quantum Schubert polynomials is equal to the coefficient of $x^{\delta}$.

Theorem 12.5. A Gromov-Witten invariant $\left\langle\sigma_{w_{1}}, \ldots, \sigma_{w_{k}}\right\rangle_{d}$ of the flag manifold is the coefficient of the monomial $q^{d} x^{\delta}$ in the normal form, with respect to the degree lexicographic order induced from $x_{1} \prec x_{2} \prec x_{3} \prec \cdots$, of the product of quantum Schubert polynomials $\mathfrak{S}_{w_{1}}^{q} \cdots \mathfrak{S}_{w_{k}}^{q}$. This normal form can be found using the reduction procedure versus the Gröbner basis $G=\left\{H_{1}^{n}, H_{2}^{n-1}, \ldots, H_{n-1}^{2}, H_{n}^{1}\right\}$.

Proof. The proof follows from Corollaries 12.2 and 12.4 and Proposition 12.1. 
We remark that it is usually more efficient to alternate normal form reduction with multiplication by $\mathfrak{S}_{w_{1}}^{q}, \mathfrak{S}_{w_{2}}^{q}, \ldots$.

The cases $k=1,2$ of Theorem 12.5 are not so interesting, since the only nonvanishing values are $\left\langle\sigma_{w_{\mathrm{o}}}\right\rangle_{(0, \ldots, 0)}=1$ and $\left\langle\sigma_{u}, \sigma_{w_{\mathrm{o}} u}\right\rangle_{(0, \ldots, 0)}=1$, for any $u \in S_{n}$. The case $k=3$ is nontrivial and actually determines all other invariants, because of the associativity property of quantum multiplication. Theorem 12.5 provides a method to directly calculate the invariants $\left\langle\sigma_{w_{1}}, \ldots, \sigma_{w_{k}}\right\rangle_{d}$ for arbitrary $k$, avoiding the use of associativity. For example - just to show the practical efficiency of the algorithms - one has, in $F l_{4}, \underbrace{\sigma_{w_{\mathrm{o}}}, \ldots, \sigma_{w_{0}}}_{17}\rangle_{(15,19,14)}=385056$.

\section{Gromov-Witten invariants For $\mathrm{Fl}_{3}$ and $\mathrm{Fl}_{4}$}

Using the method of Theorem 12.5, we calculated all 3-point Gromov-Witten invariants $\left\langle\sigma_{u}, \sigma_{v}, \sigma_{w}\right\rangle_{d}$ for the flag manifolds $\mathrm{Fl}_{3}$ and $F l_{4}$. The results are given in Tables 1 and 2, respectively. Since $\left\langle\sigma_{u}, \sigma_{v}, \sigma_{w}\right\rangle_{d}$ is invariant under permuting $u$, $v$, and $w$, each relevant unordered triple $(u, v, w)$ is listed only once. In view of the definition (2.14) of the quantum product, Tables 1 and 2 contain all information needed to construct the multiplication tables for $\mathrm{QH}\left(F l_{3}, \mathbb{Z}\right)$ and $\mathrm{QH}\left(F l_{4}, \mathbb{Z}\right)$. For example, we have $\sigma_{s_{1}} * \sigma_{w_{0}}=q_{1} \sigma_{s_{1} s_{2}}+q_{1} q_{2}$, which is a particular instance of the quantum Monk's formula (1.8). Table 1 agrees with the data obtained by P. di Francesco and C. Itzykson [10, Section 3.5] by a direct computation based on the original geometric definition.

For each triple $u, v, w \in S_{4}$, Table 2 provides reduced words for $u, v$, and $w$, and gives the polynomial $\sum_{d} q^{d}\left\langle\sigma_{u}, \sigma_{v}, \sigma_{w}\right\rangle_{d}$ unless it equals 0 . For instance, the row

\begin{tabular}{|l|l|l|l|}
\hline$u$ & $v$ & $w$ & \\
\hline 21 & 2132 & 1321 & $q_{1} q_{2}$ \\
\hline
\end{tabular}

in the table refers to the permutations $u=s_{2} s_{1}, v=s_{2} s_{1} s_{3} s_{2}$, and $w=s_{1} s_{3} s_{2} s_{1}$, and should be understood as saying that the only nonvanishing Gromov-Witten invariant $\left\langle\sigma_{u}, \sigma_{v}, \sigma_{w}\right\rangle_{d}$, for these $u, v$, and $w$, is $\left\langle\sigma_{u}, \sigma_{v}, \sigma_{w}\right\rangle_{(1,1,0)}=1$.

TABLE 1. 3-point Gromov-Witten invariants for $\mathrm{Fl}_{3}$

\begin{tabular}{|l|l|l|l|}
\hline$u$ & $v$ & $w$ & $\sum_{d} q^{d}\left\langle\sigma_{u}, \sigma_{v}, \sigma_{w}\right\rangle_{d}$ \\
\hline 1 & 1 & $w_{0}$ & 1 \\
1 & $s_{1}$ & $s_{1} s_{2}$ & 1 \\
1 & $s_{2}$ & $s_{2} s_{1}$ & 1 \\
$s_{1}$ & $s_{1}$ & $s_{2}$ & 1 \\
$s_{1}$ & $s_{2}$ & $s_{2}$ & 1 \\
$s_{1}$ & $s_{1}$ & $w_{0}$ & $q_{1}$ \\
$s_{1}$ & $s_{2} s_{1}$ & $s_{2} s_{1}$ & $q_{1}$ \\
$s_{2}$ & $s_{2}$ & $w_{0}$ & $q_{2}$ \\
$s_{2}$ & $s_{1} s_{2}$ & $s_{1} s_{2}$ & $q_{2}$ \\
$s_{1}$ & $w_{0}$ & $w_{0}$ & $q_{1} q_{2}$ \\
$s_{2}$ & $w_{0}$ & $w_{0}$ & $q_{1} q_{2}$ \\
$s_{1} s_{2}$ & $s_{2} s_{1}$ & $w_{0}$ & $q_{1} q_{2}$ \\
\hline
\end{tabular}


TABLE 2. 3-point Gromov-Witten invariants for $\mathrm{Fl}_{4}$

\begin{tabular}{|l|l|l|l|}
\hline$u$ & $v$ & $w$ & \\
\hline$\phi$ & $\phi$ & 123121 & 1 \\
$\phi$ & 1 & 12312 & 1 \\
$\phi$ & 2 & 12321 & 1 \\
$\phi$ & 3 & 21321 & 1 \\
$\phi$ & 12 & 1231 & 1 \\
$\phi$ & 21 & 1232 & 1 \\
$\phi$ & 13 & 2132 & 1 \\
$\phi$ & 23 & 1321 & 1 \\
$\phi$ & 32 & 2321 & 1 \\
$\phi$ & 123 & 121 & 1 \\
$\phi$ & 132 & 213 & 1 \\
$\phi$ & 321 & 232 & 1 \\
1 & 1 & 1232 & 1 \\
1 & 1 & 123121 & $q_{1}$ \\
1 & 2 & 1231 & 1 \\
1 & 2 & 1232 & 1 \\
1 & 3 & 2132 & 1 \\
1 & 12 & 123 & 1 \\
1 & 21 & 232 & 1 \\
1 & 21 & 12321 & $q_{1}$ \\
1 & 13 & 132 & 1 \\
1 & 13 & 232 & 1 \\
1 & 13 & 21321 & $q_{1}$ \\
1 & 23 & 121 & 1 \\
1 & 23 & 132 & 1 \\
1 & 32 & 213 & 1 \\
1 & 32 & 232 & 1 \\
1 & 121 & 1231 & $q_{1}$ \\
1 & 121 & 123121 & $q_{1} q_{2}$ \\
1 & 213 & 1321 & $q_{1}$ \\
1 & 321 & 2321 & $q_{1}$ \\
1 & 1321 & 21321 & $q_{1} q_{2}$ \\
1 & 12321 & 123121 & $q_{1} q_{2} q_{3}$ \\
2 & 2 & 1231 & 1 \\
2 & 2 & 2321 & 1 \\
2 & 2 & 123121 & $q_{2}$ \\
2 & 3 & 1321 & 1 \\
2 & 3 & 2321 & 1 \\
2 & 12 & 213 & 1 \\
2 & 12 & 12312 & $q_{2}$ \\
2 & 21 & 123 & 1 \\
2 & 21 & 232 & 1 \\
2 & 13 & 121 & 1 \\
2 & 13 & 132 & 1 \\
\hline
\end{tabular}

\begin{tabular}{|l|l|l|l|}
\hline$u$ & $v$ & $w$ & \\
\hline 2 & 13 & 213 & 1 \\
2 & 13 & 232 & 1 \\
2 & 23 & 121 & 1 \\
2 & 23 & 321 & 1 \\
2 & 32 & 213 & 1 \\
2 & 32 & 21321 & $q_{2}$ \\
2 & 121 & 1232 & $q_{2}$ \\
2 & 121 & 123121 & $q_{1} q_{2}$ \\
2 & 132 & 2132 & $q_{2}$ \\
2 & 232 & 1321 & $q_{2}$ \\
2 & 232 & 123121 & $q_{2} q_{3}$ \\
2 & 1232 & 12312 & $q_{2} q_{3}$ \\
2 & 1321 & 21321 & $q_{1} q_{2}$ \\
2 & 12321 & 123121 & $q_{1} q_{2} q_{3}$ \\
3 & 3 & 1321 & 1 \\
3 & 3 & 123121 & $q_{3}$ \\
3 & 12 & 121 & 1 \\
3 & 12 & 213 & 1 \\
3 & 21 & 132 & 1 \\
3 & 21 & 232 & 1 \\
3 & 13 & 121 & 1 \\
3 & 13 & 132 & 1 \\
3 & 13 & 12312 & $q_{3}$ \\
3 & 23 & 121 & 1 \\
3 & 23 & 12321 & $q_{3}$ \\
3 & 32 & 321 & 1 \\
3 & 123 & 1231 & $q_{3}$ \\
3 & 213 & 1232 & $q_{3}$ \\
3 & 232 & 2321 & $q_{3}$ \\
3 & 232 & 123121 & $q_{2} q_{3}$ \\
3 & 1232 & 12312 & $q_{2} q_{3}$ \\
3 & 12321 & 123121 & $q_{1} q_{2} q_{3}$ \\
12 & 12 & 13 & 1 \\
12 & 12 & 1232 & $q_{2}$ \\
12 & 21 & 23 & 1 \\
12 & 21 & 123121 & $q_{1} q_{2}$ \\
12 & 13 & 13 & 1 \\
12 & 13 & 23 & 1 \\
12 & 32 & 2132 & $q_{2}$ \\
12 & 121 & 232 & $q_{2}$ \\
12 & 121 & 12321 & $q_{1} q_{2}$ \\
12 & 132 & 132 & $q_{2}$ \\
12 & 132 & 232 & $q_{2}$ \\
12 & 321 & 21321 & $q_{1} q_{2}$ \\
\hline
\end{tabular}




\begin{tabular}{|l|l|l|l|}
\hline$u$ & $v$ & $w$ & \\
\hline 12 & 232 & 12312 & $q_{2} q_{3}$ \\
12 & 1232 & 1232 & $q_{2} q_{3}$ \\
12 & 1321 & 1321 & $q_{1} q_{2}$ \\
12 & 1321 & 2321 & $q_{1} q_{2}$ \\
12 & 2321 & 123121 & $q_{1} q_{2} q_{3}$ \\
12 & 12321 & 12321 & $q_{1} q_{2} q_{3}$ \\
12 & 123121 & 123121 & $q_{1} q_{2}^{2} q_{3}$ \\
21 & 21 & 1231 & $q_{1}$ \\
21 & 21 & 2321 & $q_{1}$ \\
21 & 13 & 32 & 1 \\
21 & 13 & 1321 & $q_{1}$ \\
21 & 13 & 2321 & $q_{1}$ \\
21 & 23 & 32 & 1 \\
21 & 121 & 213 & $q_{1}$ \\
21 & 121 & 12312 & $q_{1} q_{2}$ \\
21 & 121 & 21321 & $q_{1} q_{2}$ \\
21 & 132 & 21321 & $q_{1} q_{2}$ \\
21 & 213 & 321 & $q_{1}$ \\
21 & 1231 & 123121 & $q_{1} q_{2} q_{3}$ \\
21 & 1232 & 123121 & $q_{1} q_{2} q_{3}$ \\
21 & 2132 & 1321 & $q_{1} q_{2}$ \\
21 & 12312 & 12321 & $q_{1} q_{2} q_{3}$ \\
13 & 13 & 32 & 1 \\
13 & 13 & 1232 & $q_{3}$ \\
13 & 13 & 1321 & $q_{1}$ \\
13 & 13 & 123121 & $q_{1} q_{3}$ \\
13 & 23 & 1231 & $q_{3}$ \\
13 & 23 & 1232 & $q_{3}$ \\
13 & 32 & 32 & 1 \\
13 & 123 & 123 & $q_{3}$ \\
13 & 121 & 121 & $q_{1}$ \\
13 & 121 & 213 & $q_{1}$ \\
13 & 121 & 21321 & $q_{1} q_{2}$ \\
13 & 213 & 232 & $q_{3}$ \\
13 & 213 & 12321 & $q_{1} q_{3}$ \\
13 & 321 & 321 & $q_{1}$ \\
13 & 232 & 232 & $q_{3}$ \\
13 & 232 & 12312 & $q_{2} q_{3}$ \\
13 & 1231 & 1231 & $q_{1} q_{3}$ \\
13 & 1231 & 123121 & $q_{1} q_{2} q_{3}$ \\
13 & 1232 & 1232 & $q_{2} q_{3}$ \\
13 & 1232 & 123121 & $q_{1} q_{2} q_{3}$ \\
13 & 1321 & 1321 & $q_{1} q_{2}$ \\
13 & 1321 & 123121 & $q_{1} q_{2} q_{3}$ \\
13 & 2321 & 2321 & $q_{1} q_{3}$ \\
13 & 2321 & 123121 & $q_{1} q_{2} q_{3}$ \\
\hline
\end{tabular}

\begin{tabular}{|l|l|l|l|}
\hline$u$ & $v$ & $w$ & \\
\hline 13 & 12312 & 12321 & $q_{1} q_{2} q_{3}$ \\
13 & 12321 & 21321 & $q_{1} q_{2} q_{3}$ \\
23 & 23 & 1231 & $q_{3}$ \\
23 & 23 & 2321 & $q_{3}$ \\
23 & 32 & 123121 & $q_{2} q_{3}$ \\
23 & 123 & 213 & $q_{3}$ \\
23 & 132 & 12312 & $q_{2} q_{3}$ \\
23 & 213 & 232 & $q_{3}$ \\
23 & 232 & 12312 & $q_{2} q_{3}$ \\
23 & 232 & 21321 & $q_{2} q_{3}$ \\
23 & 1232 & 2132 & $q_{2} q_{3}$ \\
23 & 1321 & 123121 & $q_{1} q_{2} q_{3}$ \\
23 & 2321 & 123121 & $q_{1} q_{2} q_{3}$ \\
23 & 12321 & 21321 & $q_{1} q_{2} q_{3}$ \\
32 & 32 & 1321 & $q_{2}$ \\
32 & 123 & 12312 & $q_{2} q_{3}$ \\
32 & 121 & 132 & $q_{2}$ \\
32 & 121 & 232 & $q_{2}$ \\
32 & 121 & 21321 & $q_{1} q_{2}$ \\
32 & 132 & 132 & $q_{2}$ \\
32 & 232 & 12321 & $q_{2} q_{3}$ \\
32 & 1231 & 1232 & $q_{2} q_{3}$ \\
32 & 1231 & 123121 & $q_{1} q_{2} q_{3}$ \\
32 & 1232 & 1232 & $q_{2} q_{3}$ \\
32 & 1321 & 1321 & $q_{1} q_{2}$ \\
32 & 12321 & 12321 & $q_{1} q_{2} q_{3}$ \\
32 & 123121 & 123121 & $q_{1} q_{2}^{2} q_{3}$ \\
123 & 132 & 1232 & $q_{2} q_{3}$ \\
123 & 321 & 123121 & $q_{1} q_{2} q_{3}$ \\
123 & 232 & 2132 & $q_{2} q_{3}$ \\
123 & 1321 & 12321 & $q_{1} q_{2} q_{3}$ \\
123 & 2321 & 21321 & $q_{1} q_{2} q_{3}$ \\
121 & 121 & 1231 & $q_{1} q_{2}$ \\
121 & 121 & 1232 & $q_{1} q_{2}$ \\
121 & 121 & 1321 & $q_{1} q_{2}$ \\
121 & 121 & 2321 & $q_{1} q_{2}$ \\
121 & 132 & 1321 & $q_{1} q_{2}$ \\
121 & 132 & 2321 & $q_{1} q_{2}$ \\
121 & 213 & 1321 & $q_{1} q_{2}$ \\
121 & 213 & 123121 & $q_{1} q_{2} q_{3}$ \\
121 & 321 & 2132 & $q_{1} q_{2}$ \\
121 & 232 & 1232 & $q_{2} q_{3}$ \\
121 & 232 & 1321 & $q_{1} q_{2}$ \\
121 & 232 & 123121 & $q_{1} q_{2} q_{3}$ \\
121 & 1231 & 12321 & $q_{1} q_{2} q_{3}$ \\
121 & 1232 & 12321 & $q_{1} q_{2} q_{3}$ \\
\hline
\end{tabular}




\begin{tabular}{|l|l|l|l|}
\hline$u$ & $v$ & $w$ & \\
\hline 121 & 2321 & 12312 & $q_{1} q_{2} q_{3}$ \\
121 & 12312 & 123121 & $q_{1} q_{2}^{2} q_{3}$ \\
132 & 213 & 123121 & $q_{1} q_{2} q_{3}$ \\
132 & 321 & 1321 & $q_{1} q_{2}$ \\
132 & 232 & 1231 & $q_{2} q_{3}$ \\
132 & 232 & 1232 & $q_{2} q_{3}$ \\
132 & 1231 & 12321 & $q_{1} q_{2} q_{3}$ \\
132 & 2321 & 12321 & $q_{1} q_{2} q_{3}$ \\
132 & 12312 & 123121 & $q_{1} q_{2}^{2} q_{3}$ \\
132 & 21321 & 123121 & $q_{1} q_{2}^{2} q_{3}$ \\
213 & 213 & 1231 & $q_{1} q_{3}$ \\
213 & 213 & 2321 & $q_{1} q_{3}$ \\
213 & 213 & 123121 & $q_{1} q_{2} q_{3}$ \\
213 & 232 & 1232 & $q_{2} q_{3}$ \\
213 & 232 & 123121 & $q_{1} q_{2} q_{3}$ \\
213 & 1231 & 21321 & $q_{1} q_{2} q_{3}$ \\
213 & 1232 & 21321 & $q_{1} q_{2} q_{3}$ \\
213 & 2132 & 12321 & $q_{1} q_{2} q_{3}$ \\
213 & 1321 & 12312 & $q_{1} q_{2} q_{3}$ \\
213 & 2321 & 12312 & $q_{1} q_{2} q_{3}$ \\
321 & 1231 & 12312 & $q_{1} q_{2} q_{3}$ \\
321 & 1232 & 12321 & $q_{1} q_{2} q_{3}$ \\
232 & 232 & 1231 & $q_{2} q_{3}$ \\
\hline
\end{tabular}

\begin{tabular}{|l|l|l|l|}
\hline$u$ & $v$ & $w$ & \\
\hline 232 & 232 & 1232 & $q_{2} q_{3}$ \\
232 & 232 & 1321 & $q_{2} q_{3}$ \\
232 & 232 & 2321 & $q_{2} q_{3}$ \\
232 & 1231 & 21321 & $q_{1} q_{2} q_{3}$ \\
232 & 1321 & 12321 & $q_{1} q_{2} q_{3}$ \\
232 & 2321 & 12321 & $q_{1} q_{2} q_{3}$ \\
232 & 21321 & 123121 & $q_{1} q_{2}^{2} q_{3}$ \\
1231 & 1231 & 1321 & $q_{1} q_{2} q_{3}$ \\
1231 & 1232 & 1321 & $q_{1} q_{2} q_{3}$ \\
1231 & 2132 & 2321 & $q_{1} q_{2} q_{3}$ \\
1232 & 1321 & 2321 & $q_{1} q_{2} q_{3}$ \\
1232 & 1321 & 123121 & $q_{1} q_{2}^{2} q_{3}$ \\
1232 & 2321 & 2321 & $q_{1} q_{2} q_{3}$ \\
1232 & 21321 & 21321 & $q_{1} q_{2}^{2} q_{3}$ \\
1232 & 123121 & 123121 & $q_{1} q_{2}^{2} q_{3}^{2}$ \\
2132 & 2132 & 123121 & $q_{1} q_{2}^{2} q_{3}$ \\
2132 & 12312 & 21321 & $q_{1} q_{2}^{2} q_{3}$ \\
1321 & 12312 & 12312 & $q_{1} q_{2}^{2} q_{3}$ \\
1321 & 123121 & 123121 & $q_{1}^{2} q_{2}^{2} q_{3}$ \\
12312 & 12312 & 123121 & $q_{1} q_{2}^{2} q_{3}^{2}$ \\
21321 & 21321 & 123121 & $q_{1}^{2} q_{2}^{2} q_{3}$ \\
123121 & 123121 & 123121 & $q_{1}^{2} q_{2}^{2} q_{3}^{2}$ \\
& & & \\
\hline
\end{tabular}

14. Tables of quantum Schubert polynomials

$$
n=2
$$

\begin{tabular}{|l|l|l|l|}
\hline$w$ & red.word & $\mathfrak{S}_{w}$ & $\mathfrak{S}_{w}^{q}$ \\
\hline 12 & $\phi$ & $e_{0}$ & 1 \\
21 & 1 & $e_{1}$ & $x_{1}$ \\
\hline
\end{tabular}

$$
n=3
$$

\begin{tabular}{|l|l|l|l|}
\hline$w$ & red.word & $\mathfrak{S}_{w}$ & $\mathfrak{S}_{w}^{q}$ \\
\hline 123 & $\phi$ & $e_{00}$ & 1 \\
213 & 1 & $e_{10}$ & $x_{1}$ \\
132 & 2 & $e_{01}$ & $x_{1}+x_{2}$ \\
231 & 12 & $e_{02}$ & $x_{1} x_{2}+q_{1}$ \\
312 & 21 & $e_{11}-e_{02}$ & $x_{1}^{2}-q_{1}$ \\
321 & 121 & $e_{12}$ & $x_{1}\left(x_{1} x_{2}+q_{1}\right)$ \\
\hline
\end{tabular}




$$
n=4
$$

\begin{tabular}{|c|c|c|c|}
\hline$w$ & red.word & $\mathfrak{S}_{w}$ & $\mathfrak{S}_{w}^{q}$ \\
\hline 1234 & $\phi$ & $e_{000}$ & 1 \\
\hline 2134 & 1 & $e_{100}$ & $x_{1}$ \\
\hline 1324 & 2 & $e_{010}$ & $x_{1}+x_{2}$ \\
\hline 1243 & 3 & $e_{001}$ & $x_{1}+x_{2}+x_{3}$ \\
\hline 2314 & 12 & $e_{020}$ & $x_{1} x_{2}+q_{1}$ \\
\hline 3124 & 21 & $e_{110}-e_{020}$ & $x_{1}^{2}-q_{1}$ \\
\hline 2143 & 13 & $e_{101}$ & $x_{1}\left(x_{1}+x_{2}+x_{3}\right)$ \\
\hline 1342 & 23 & $e_{002}$ & $x_{1} x_{2}+x_{1} x_{3}+x_{2} x_{3}+q_{1}+q_{2}$ \\
\hline 1423 & 32 & $e_{011}-e_{002}$ & $x_{1}^{2}+x_{1} x_{2}+x_{2}^{2}-q_{1}-q_{2}$ \\
\hline 2341 & 123 & $e_{003}$ & $x_{1} x_{2} x_{3}+q_{1} x_{3}+q_{2} x_{1}$ \\
\hline 3214 & 121 & $e_{120}$ & $x_{1}\left(x_{1} x_{2}+q_{1}\right)$ \\
\hline 2413 & 132 & $e_{021}-e_{003}$ & $x_{1}^{2} x_{2}+x_{1} x_{2}^{2}+q_{1} x_{1}+q_{1} x_{2}-q_{2} x_{1}$ \\
\hline 3142 & 213 & $e_{102}-e_{003}$ & $x_{1}^{2} x_{2}+x_{1}^{2} x_{3}+q_{1} x_{1}-q_{1} x_{3}$ \\
\hline 4123 & 321 & $\begin{aligned} e_{111} & -e_{021} \\
& -e_{021}+e_{003}\end{aligned}$ & $x_{1}^{3}-2 q_{1} x_{1}-q_{1} x_{2}$ \\
\hline 1432 & 232 & $e_{012}-e_{003}$ & $\begin{aligned} x_{1}^{2} x_{2} & +x_{1}^{2} x_{3}+x_{1} x_{2}^{2}+x_{1} x_{2} x_{3}+x_{2}^{2} x_{3} \\
& +q_{1} x_{1}+q_{1} x_{2}-q_{1} x_{3}+q_{2} x_{2}\end{aligned}$ \\
\hline 3241 & 1231 & $e_{103}$ & $x_{1}\left(x_{1} x_{2} x_{3}+q_{1} x_{3}+q_{2} x_{1}\right)$ \\
\hline 2431 & 1232 & $e_{013}$ & $\left(x_{1}+x_{2}\right)\left(x_{1} x_{2} x_{3}+q_{1} x_{3}+q_{2} x_{1}\right)$ \\
\hline 3412 & 2132 & $e_{022}-e_{013}$ & $x_{1}^{2} x_{2}^{2}+2 q_{1} x_{1} x_{2}-q_{2} x_{1}^{2}+q_{1}^{2}+q_{1} q_{2}$ \\
\hline 4213 & 1321 & $\begin{array}{l}e_{121}-e_{022} \\
\quad+e_{013}-e_{103}\end{array}$ & $x_{1}^{3} x_{2}+q_{1} x_{1}^{2}-q_{1} x_{1} x_{2}-q_{1}^{2}-q_{1} q_{2}$ \\
\hline 4132 & 2321 & $e_{112}-e_{022}-e_{103}$ & $\begin{aligned} x_{1}^{3} x_{2} & +x_{1}^{3} x_{3}+q_{1} x_{1}^{2}-q_{1} x_{1} x_{2} \\
& -2 q_{1} x_{1} x_{3}-q_{1} x_{2} x_{3}-q_{1}^{2}-q_{1} q_{2}\end{aligned}$ \\
\hline 3421 & 12312 & $e_{023}$ & $\left(x_{1} x_{2}+q_{1}\right)\left(x_{1} x_{2} x_{3}+q_{1} x_{3}+q_{2} x_{1}\right)$ \\
\hline 4231 & 12321 & $e_{113}-e_{023}$ & $\left(x_{1}^{2}-q_{1}\right)\left(x_{1} x_{2} x_{3}+q_{1} x_{3}+q_{2} x_{1}\right)$ \\
\hline 4312 & 21321 & $e_{122}-e_{113}$ & $x_{1}\left(x_{1}^{2} x_{2}^{2}+2 q_{1} x_{1} x_{2}-q_{2} x_{1}^{2}+q_{1}^{2}+q_{1} q_{2}\right)$ \\
\hline 4321 & 123121 & $e_{123}$ & $x_{1}\left(x_{1} x_{2}+q_{1}\right)\left(x_{1} x_{2} x_{3}+q_{1} x_{3}+q_{2} x_{1}\right)$ \\
\hline
\end{tabular}

\section{ACKNOWLEDGMENTS}

The authors are grateful to Alexander Astashkevich, Alexander Givental, Alain Lascoux, and Yuri Manin for stimulating discussions and helpful remarks. Several inaccuracies in the original version were corrected by the referee. Our special gratitude goes to William Fulton for thoughtful comments and suggestions, which led to substantial improvements of the paper.

The preliminary version of this paper appeared as [12].

\section{REFERENCES}

1. A. Astashkevich and V. Sadov, Quantum cohomology of partial flag manifolds $F_{n_{1}, \ldots, n_{k}}$, Comm. Math. Phys. 170 (1995), 503-528. MR 96g:58027

2. I. N. Bernstein, I. M. Gelfand, and S. I. Gelfand, Schubert cells and cohomology of the space $G / P$, Russian Math. Surveys 28 (1973), 1-26. MR 55:2941 
3. A. Bertram, Quantum Schubert calculus, Advances in Math. (to appear).

4. S. C. Billey and M. Haiman, Schubert polynomials for the classical groups, J. Amer. Math. Soc. 8 (1995), 443-482. CMP 95:05

5. A. Borel, Sur la cohomologie des espaces fibrés principaux et des espaces homogènes des groupes de Lie compacts, Ann. of Math. (2) 57 (1953), 115-207. MR 14:490e

6. C. Chevalley, Sur les décompositions cellulaires des espaces $G / B$, in: Algebraic Groups and Their Generalizations (W. Haboush and B. Parshall, eds.), Proc. Symp. Pure Math., vol. 56, Part 1, Amer. Math. Soc., Providence, RI, 1994, pp. 1-23. MR 95e:14041

7. I. Ciocan-Fontanine, Quantum cohomology of flag varieties, Intern. Math. Research Notices (1995), no. 6, 263-277. MR 96h:14071

8. M. Demazure, Désingularization des variétés de Schubert généralisées, Ann. Scient. Ecole Normale Sup. (4) 7 (1974), 53-88. MR 50:7174

9. C. Ehresmann, Sur la topologie de certains espaces homogènes, Ann. of Math. 35 (1934), 396-443.

10. P. di Francesco and C. Itzykson, Quantum intersection rings, in: The Moduli Space of Curves (R. Dijkgraaf, C. Faber, and G. van der Geer, eds.), Progress in Mathematics, vol. 129, Birkhäuser, Boston, 1995, pp. 81-148. MR 96k:14041

11. S. Fomin and A. N. Kirillov, Combinatorial $B_{n}$-analogues of Schubert polynomials, Trans. Amer. Math. Soc. 348 (1996), no. 9, 3591-3620. CMP 96:15

12. S. Fomin, S. Gelfand, and A. Postnikov, Quantum Schubert polynomials, AMS electronic preprint AMSPPS \#199605-14-008, April 1996.

13. W. Fulton, Young tableaux with applications to representation theory and geometry, Cambridge University Press, 1996.

14. W. Fulton and R. Pandharipande, Notes on stable maps and quantum cohomology, preprint alg-geom/9608011.

15. A. Givental and B. Kim, Quantum cohomology of flag manifolds and Toda lattices, Comm. Math. Phys. 168 (1995), 609-641. MR 96c:58027

16. B. Kim, Quantum cohomology of partial flag manifolds and a residue formula for their intersection pairing, Intern. Math. Research Notices (1995), no. 1, 1-16. MR 96c:58028

17. B. Kim, On equivariant quantum cohomology, Intern. Math. Research Notices (1996), no. 17, 841-851. CMP 97:04

18. B. Kim, Quantum cohomology of flag manifolds $G / B$ and quantum Toda lattices, preprint alg-geom $/ 9607001$.

19. M. Kontsevich and Yu. Manin, Gromov-Witten classes, quantum cohomology, and enumerative geometry, Comm. Math. Phys. 164 (1994), 525-562. MR 95i:14049

20. B. Kostant, Flag manifold quantum cohomology, the Toda lattice, and the representation with highest weight $\rho$, Selecta Math. (N.S.) 2 (1996), 43-91. CMP 96:16

21. A. Lascoux, Classes de Chern des varietes de drapeaux, C. R. Acad. Sci. Paris 295 (1982), 393-398. MR 85e:14074

22. A. Lascoux and M. P. Schützenberger, Polynômes de Schubert, C. R. Acad. Sci. Paris 294 (1982), 447-450. MR 83e:14039

23. A. Lascoux and M. P. Schützenberger, Fonctorialité de polynômes de Schubert, Contemp. Math. 88 (1989), 585-598. MR 90i:16003

24. J. Li and G. Tian, The quantum cohomology of homogeneous varieties, J. Algebraic Geom. (to appear).

25. I. G. Macdonald, Notes on Schubert polynomials, Publications LACIM, Montréal, 1991.

26. D. Monk, The geometry of flag manifolds, Proc. London Math. Soc. 9 (1959), 253-286. MR 21:5641

27. P. Pragacz and J. Ratajski, Formulas for Lagrangian and orthogonal degeneracy loci; the Q-polynomial approach, Compositio Math. (to appear).

28. Y. Ruan and G. Tian, Mathematical theory of quantum cohomology, J. Diff. Geom. 42 (1995), no. 2, 259-367. MR 96m:58033

29. B. Sturmfels, Algorithms in invariant theory, Springer-Verlag, Berlin, 1993. MR 94m:13004

30. C. Vafa, Topological mirrors and quantum rings, in: Essays on Mirror Manifolds (S.-T. Yau, ed.), International Press, Boston, 1992. MR 94c:81193

31. E. Witten, Two-dimensional gravity and intersection theory on moduli space, Surveys in Differential Geometry, vol. 1, International Press, Boston, 1991, pp. 243-310. MR 93e:32028 
Department of Mathematics, Massachusetts Institute of Technology, Cambridge, MASSACHUSETTS 02139

E-mail address: fomin@math.mit.edu

American Mathematical Society, P.O.Box 6248, Providence, Rhode Island 02940-6248

E-mail address: sxg@ams.org

Department of Mathematics, Massachusetts Institute of Technology, Cambridge, MASSACHUSETTS 02139

E-mail address: apost@math.mit.edu 